\title{
HOMOCLINIC BIFURCATIONS AND UNIFORM HYPERBOLICITY FOR THREE-DIMENSIONAL FLOWS
}

\section{BIFURCATIONS HOMOCLINES ET HYPERBOLICITÉ UNIFORME POUR LES FLOTS TRIDIMENSIONELS}

\author{
Aubin ARROYO ${ }^{\mathrm{a}, *, 1}$, Federico RODRIGUEZ HERTZ ${ }^{\mathrm{b}, 2}$ \\ a Instituto de Matemáticas, UNAM (Unidad Cuernavaca) Universidad Nacional Autónoma de México, \\ A.P. 273 Admon de correos \# 3 C.P. 62251, Cuernavaca Morelos, Mexico \\ ${ }^{\mathrm{b}}$ Facultad de Ingeniería (IMERL) Av. Julio Herrera y Reissig 565 CP 11300, Montevideo, Uruguay
}

Received 18 July 2002, accepted 26 September 2002

ABSTRACT. - In this paper we prove that any $C^{1}$ vector field defined on a three-dimensional manifold can be approximated by one that is uniformly hyperbolic, or that exhibits either a homoclinic tangency or a singular cycle. This proves an analogous statement of a conjecture of Palis for diffeomorphisms in the context of $C^{1}$-flows on three manifolds. For that, we rely on the notion of dominated splitting for the associated linear Poincaré flow.

(C) 2003 L'Association Publications de l'Institut Henri Poincaré. Published by Elsevier B.V. All rights reserved

RÉSUMÉ. - On prouve que tout champ de vecteurs $C^{1}$ défini sur une variété de dimension trois peut être approché par un qui est uniformément hyperbolique ou bien par un qui présente soit une tangence homocline soit un cycle singulier. Ceci prouve, dans le contexte des flots $C^{1}$ sur les variétés de dimension trois, l'analogue d'une conjecture de Palis concernant les difféomorphismes. On s'appuie sur la notion de décomposition dominée pour le flot linéaire de Poincaré associé.

(C) 2003 L'Association Publications de l'Institut Henri Poincaré. Published by Elsevier B.V. All rights reserved

\section{Introduction}

The quest to understand the asymptotic behavior of orbits of a large set of evolution laws has been guiding one important strain of the theory of dynamical systems. In the sixties the notion of structural stable systems, first introduced by Andronov and

\footnotetext{
* Corresponding author.

E-mail addresses: aubin@matcuer.unam.mx (A. Arroyo), frhertz@ fing.edu.uy (F. Rodriguez Hertz).

${ }^{1}$ Partially supported by CNPq-Brazil and CONACYT-México.

2 Partially supported by CNPq-Brazil.
} 
Pontryagin thirty years before, meaning systems that are equivalent up to a continuous global change of coordinates to all nearby ones, was at the center of the attempts to provide a typical model in the universe of dynamical systems.

In a series of papers written in that decade and in early seventies, Anosov, Palis and Smale, Robbin, de Melo and Robinson, prove that uniform hyperbolicity and transversality of stable and unstable manifolds imply structural stability. In the mideighties, Mañé obtained the converse statement for diffeomorphisms, in a profound and very original work. Ten years later, Hayashi extended this result for flows. This constitutes a remarkable page in the history of modern dynamics: The solution of the Stability Conjecture, [16].

Recall that an invariant set $\Lambda$ of a flow $X$ is hyperbolic if its tangent bundle splits into three $D X$-invariant sub-bundles, $E^{s}, E^{u}$ and $[X]$, where vectors on $E^{s}$ are uniformly contracted in the future, the same for $E^{u}$ in the past, and $[X]$ is the flow direction. A flow $X$ is uniformly hyperbolic (or Axiom A) if its limit set is hyperbolic. For the case of diffeomorphisms this concept is similar by considering only the first two subbundles. For a complete description of uniform hyperbolicity see [17] and [24]. Yet and remarkably so, at the second half of the sixties it was already clear that uniform hyperbolicity could not be present for every system of a dense subset in the universe of all dynamics.

A key question was then: Is it possible to look for a general scenario for dynamics? The search for such an answer, in particular the work of Newhouse on the coexistence of infinitely many sinks (see [10]), draw the attention to homoclinic orbits. That is, orbits that in the past and the future converge to the same periodic orbit, which has been first considered by Poincaré almost a century before. Altogether, the examples by Newhouse gave rise to a rich family of new dynamics. The creation-destruction of such orbits is, roughly speaking, what its meant by homoclinic bifurcations; see [17] for a formal definition. Based on these and other subsequent developments, Palis formulated in the mid-nineties the following conjecture (see [17] and [14]):

CONJECTURE 1. - The diffeomorphisms exhibiting a homoclinic bifurcation are $C^{r}$ dense in the complement of the closure of the hyperbolic ones $(r \geqslant 1)$.

There has been substantial advances in this direction. The work of Pujals and Sambarino [21] is an important example: They provided a proof of the conjecture in the case of diffeomorphisms defined on a compact surface in the $C^{1}$ topology.

In the setting of diffeomorphisms on two-dimensional manifolds, homoclinic bifurcations arise from homoclinic tangencies. Recall that the stable and unstable manifolds of a hyperbolic periodic point $p, W^{s}(p)$ and $W^{u}(p)$, respectively, are immersed submanifolds on the ambient space. A point $q \in W^{s}(p) \cap W^{u}(p)$ is a homoclinic point if $q \neq p$. If these submanifols meet tangentialy at a homoclinic point $q$, we say such point is a homoclinic tangency.

Dealing with the above conjecture in the context of flows, another homoclinic phenomenon involving singularities of the vector field must be considered: singular cycles. Recall that a singular point $\sigma$ of a vector field $X$ is hyperbolic if the derivative $D_{\sigma} X$ has no eigenvalues which are purely imaginary, and in this case we have stable and unstable manifolds of $\sigma$, denoted by $W^{s}(\sigma)$, and $W^{u}(\sigma)$, respectively. It may happen 
that $q \in W^{s}(\sigma) \cap W^{u}(\sigma)$ for $q \neq \sigma$. In this case we say the vector field has a singular cycle, a concept that was first considered by Labarca and Pacífico [7]. In dimension three, these are all possible homoclinic bifurcations: homoclinic tangencies and singular cycles.

This work is intended to give a positive answer to the conjecture above, in the context of three-dimensional vector fields and the $C^{1}$-topology. To be more precise, let $M$ be a compact manifold of dimension three without boundary. Denote by $\mathcal{X}^{r}(M)$ the space of vector fields of class $C^{r}(r \geqslant 1)$ defined on $M$.

THEOREM A. - Any vector field $X \in \mathcal{X}^{1}(M)$ can be approximated by another one $Y \in \mathcal{X}^{1}(M)$ showing one of the following phenomena:

(1) Uniform hyperbolicity with the no-cycles condition.

(2) A homoclinic tangency.

(3) A singular cycle.

As in the case of surface diffeomorphisms, our methods rely on a relaxed form of hyperbolicity, first used by Mañé, Liao and Pliss in their attempts to characterize structural stable diffeomorphisms. It is called dominated splitting and it strictly includes the class partially hyperbolic systems (see [6], [1] and [25]). An invariant set $\Lambda$ for a diffeomorphism $f$ has a dominated splitting if its tangent bundle decomposes into two $D f$-invariant sub-bundles $T_{\Lambda} M=E \oplus F$ and there are constants $C>0$ and $\lambda \in(0,1)$ such that for all $x \in \Lambda$ :

$$
\left\|\left.D f^{n}\right|_{E(x)}\right\|\left\|\left.D f^{-n}\right|_{F\left(f^{n}(x)\right)}\right\|<C \lambda^{n} ; \quad \forall n \geqslant 0 .
$$

There is a remarkable relationship between the absence of homoclinic tangencies and the existence of a dominated splitting. This fact is explored in [21] and more recently, the same authors provided a rather complete description of the dynamics underlying a dominated splitting for surface diffeomorphisms on [22].

Still, the natural translation of this notion into the context of three-dimensional flows is more limited. That is, a splitting of the kind: $T_{\Lambda} M=E \oplus[X] \oplus F$, where each sub-bundle is one-dimensional, or $T_{\Lambda} M=E \oplus F$, where one is one-dimensional, both exclude the scenarios depicted in the following example:

Consider an Anosov flow $A$ on $M$ and choose an infinite sequence of periodic orbits with periods arbitrarily large, say $\left\{p_{n}\right\}$. An application of Frank's Lemma (see [9] for a flow version) allows us to perturb $\left(C^{1}\right)$ the flow on each $p_{n}$ by pushing the strongstable direction over the tangent direction of the orbit of $p_{n}$ within the central stable space defined by $E^{s}+[X]$. In the same way, in another part of the orbit of $p_{n}$, we push the strong-unstable direction. Inductively, this process renders a sequence $X_{n}$ of vector fields. Observe that no limit of $\left\{X_{n}\right\}$ can have an invariant splitting of any of the kind as above. Also it is not possible to find a nearby flow exhibiting a homoclinic tangency, since strong directions remain orthogonal to each other on each point.

Like hyperbolicity, the property of a dominated splitting is a condition on the action of the derivative on the tangent bundle, which determines some dynamical behavior of the transformation itself. In our case, we shall consider a condition not on the whole tangent bundle but on its projection over a certain sub-bundle everywhere normal to the flow 
direction, to which the linear Poincare flow is associated. We shall be precise about this in the next section. However, such notion of dominated splitting for the linear Poincare flow can be obtained once we are far from homoclinic tangencies (see Theorem 6.2 for a precise statement).

On the other hand, Theorem A relies on a fundamental result related to the possible underlying dynamics of an invariant set provided with a dominated splitting. To explain this, let us call an embedded two-dimensional torus $\mathcal{T} \subset M$ an irrational torus if it is invariant by the action of $X^{t}$, it is normally hyperbolic and moreover, $\left.X^{t}\right|_{\mathcal{T}}$ is conjugated to a linear irrational flow on $S^{1} \times S^{1}$. Denote by $\operatorname{Per}(X)$ the set of periodic orbits of $X$, and $\operatorname{Sing}(X)=\{p \in M \mid X(p)=0\}$, for a given $X \in \mathcal{X}^{r}(M), M$ being a closed threemanifold.

THEOREM B. - Let $\Lambda$ be a compact invariant set for $X \in \mathcal{X}^{2}(M)$ with a dominated splitting and such that $\operatorname{Sing}(X) \cap \Lambda=\emptyset$ and all points in $\operatorname{Per}(X) \cap \Lambda$ are hyperbolic saddles, then $\Lambda=\tilde{\Lambda} \cup \mathcal{T}$, where $\tilde{\Lambda}$ is hyperbolic and $\mathcal{T}$ is a finite union of irrational tori.

The dominated splitting provides us with invariant foliations over the invariant set; formed by central stable and central unstable manifolds (see Lemma 3.2). A priori, such leaves do not have a dynamical meaning at all. However, a kind of Denjoy's property asserts that smoothness of the vector field does not allow the existence of wandering intervals; moreover, it provides us the asymptotic behavior of local central manifolds. This allows us to construct actual stable and unstable manifolds from the central ones (see Proposition 4.2 and Lemma 4.14). For that, we must establish a way to control how fast orbits get farther apart as we iterate; not as two points at certain flow-time but as orbits of the linear Poincare flow. In order to do that, it ought to be necessary to consider a set of holonomy maps defined on transversal two-dimensional sections. These maps together work as a kind of global Poincaré transformation and allow us to proceed, at least locally, as in the case of a surface-diffeomorphism (see Section 2.2). In fact, most of our effort will be spent in order to achieve such setting. After that, some modifications of the arguments of [21] are needed to conclude the proof of Theorem B. Indeed, [21] is a key reference throughout this paper.

Further questions. Lorenz-like attractors (see [3]) are a kind of rich dynamics that can appear from singular cycles. They are characterized by the presence of infinitely many periodic orbits in any neighborhood of a singularity, in a robust way. The study of this situation lead Morales, Pacífico and Pujals to define an adequate notion of hyperbolicity, where singularities are involved, called singular hyperbolicity (see [9]).

This suggest a stronger statement of Theorem A, replacing Singular Cycle by Singular Hyperbolic, on item (3); and a proof will certainly rely on an application of their methods. However, some work has to be done before, in particular on the choice of the dominated splitting over an invariant set which contains a singularity, since the linear Poincaré flow it is not defined on equilibrium points. Nevertheless, it seems to be useful to consider also a splitting on the tangent bundle as in the way is done for singular hyperbolic sets.

The paper is structured as follows: In Section 2 we set up a useful notion of dominated splitting for invariant sets, related to the linear Poincaré flow. In Section 3 we construct 
central manifolds for sets with dominated splitting. The proof of Theorem B will be there reduced to a Main Lemma. Section 4 is for proving dynamical properties of central manifolds. This is the kernel of this work. Section 5 is devoted to give a proof for the Main Lemma and finally, in Section 6 we prove Theorem A.

\section{Dominated splitting for invariant sets}

This section is devoted to construct a useful notion of dominated splitting for invariant sets for flows. For that, we shall consider the linear Poincaré flow which acts on certain sub-bundle of the tangent space. At the end of this section, we shall obtain a family of holonomy maps defined between pairs of two-dimensional sections each one transversal to the flow. This family of maps acts on the manifold in the same way the linear Poincaré flow acts on the normal bundle.

Let $M$ be a compact three-dimensional riemannian manifold without boundary. Denote by $X^{t}$ the flow associated to $X \in \mathcal{X}^{1}(M)$, that is, a family of diffeomorphisms on $M$ such that $X^{s} \circ X^{t}=X^{s+t}$ for all $s, t \in \mathbb{R}, X^{0}=\mathrm{Id}$ and $\mathrm{d} X^{t}(p) /\left.\mathrm{d} t\right|_{t=0}=X(p)$, for any $p \in M$.

\subsection{The linear Poincaré flow}

The vector field $X$ induces two sub-bundles on $T_{Q} M$, say, $[X]_{p}=\left\{v \in T_{p} M \mid v=\right.$ $\alpha X(p), \alpha \in \mathbb{R}\}$ and $\tilde{\mathcal{N}}_{p}=[X]_{p}^{\perp}$, for any $p \notin \operatorname{Sing}(X)$. Denote by $\mathcal{N}=\frac{T_{Q} M}{[X]}$ the normal bundle of $X$ over $Q=M \backslash \operatorname{Sing}(X)$, the regular points of $X$. For each $t \in \mathbb{R}$ the tangent map of $X^{t}$ restricted to $\mathcal{N} \subset T_{Q} M$ induces an automorphism on $\mathcal{N}$ that commutes the following diagram:

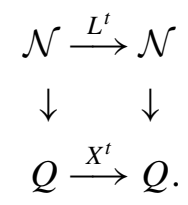

The flow of automorphisms $\left\{L^{t}\right\}_{t \in \mathbb{R}}$ is called the linear Poincare flow of the normal bundle $\mathcal{N}$. We took this notion from the work of Doering, [2], and it allows us to define an adequate notion of dominated splitting for invariant sets of flows.

Definition 1.- An invariant set $\Lambda$ has a dominated splitting if there exists a splitting of the normal bundle of $\tilde{\Lambda}=\Lambda \backslash \operatorname{Sing}(X)$, say $\mathcal{N}_{\tilde{\Lambda}}=E \oplus F$ and there are numbers $C>0$ and $\lambda \in(0,1)$ such that: $L^{t}(E(x))=E\left(X^{t}(x)\right), L^{t}(F(x))=F\left(X^{t}(x)\right)$ and

$$
\left.\left|L^{t}\right|_{E(x)}|| L^{-t}\right|_{F\left(X^{t}(x)\right)} \mid \leqslant C \lambda^{t} ; \quad \forall t \geqslant 0 .
$$

Fix an invariant set $\Lambda$ for $X^{t}$ and take a splitting of $\mathcal{N}_{\tilde{\Lambda}}=E^{\prime} \oplus F^{\prime}$, not necessarily invariant. For any number $a \in(0,1]$ we can define the following two cone fields pointwise. On each $x \in \tilde{\Lambda}$ set: 


$$
\begin{aligned}
& C_{a}^{E}(x)=\left\{w \in \mathcal{N}_{x}\left|w=v_{E}+v_{F},\right| v_{F}|\leqslant a| v_{E} \mid\right\}, \\
& C_{a}^{F}(x)=\left\{w \in \mathcal{N}_{x}\left|w=v_{E}+v_{F},\right| v_{E}|\leqslant a| v_{F} \mid\right\} .
\end{aligned}
$$

As in the hyperbolic case, it is not difficult to see that if this cone field is invariant under the action of $L^{t}$ we obtain a dominated splitting on $\Lambda$ (see [12]). The following lemma states this.

LEMMA 2.1. - If there exist $\lambda<1, a>0$, and there is a splitting of $\mathcal{N}_{\Lambda}=E^{\prime} \oplus F^{\prime}$ such that

$$
L^{t}\left(C_{a}^{F^{\prime}}\right) \subset C_{\lambda^{t} a}^{F^{\prime}}\left(X^{t}(x)\right) \quad \text { and } \quad L^{-t}\left(C_{a}^{E^{\prime}}\right) \subset C_{\lambda^{t} a}^{E^{\prime}}\left(X^{-t}(x)\right)
$$

for all $t \geqslant 0$, then $\Lambda$ has a dominated splitting.

Moreover, the following lemma due to Doering (see Proposition 1.1 in [2]), asserts that in the case of hyperbolicity, these two notions are coherent.

LEMMA 2.2. - Let $\Lambda \subset M$ an invariant compact set for $X^{t} . \Lambda$ has a hyperbolic structure for $X^{t}$ if and only if the linear Poincaré flow restricted over $\Lambda$ is hyperbolic.

\subsection{Holonomy maps}

Let $\Lambda \subset M$ be a compact invariant set with dominated splitting such that $\Lambda \cap$ $\operatorname{Sing}(X)=\emptyset$. Given $a \in(0,1]$ let $U_{a}$ be a neighborhood of $\Lambda$ in $\mathcal{N}$, where is defined an invariant $a$-cone field. Denote by $\mathcal{N}_{x}(\varepsilon)$ the ball of radius $\varepsilon>0$ on $\mathcal{N}_{x}$. Notice that there exists a neighborhood $K$ such that

$$
\Lambda \subset \operatorname{int}(K) \subset \operatorname{cl}(K) \subset U_{1} \subset M \backslash \operatorname{Sing}(X)
$$

since $\operatorname{Sing}(X)$ is a closed set. Hence, there is $\varepsilon^{*}>0$ such that the exponential map $\exp _{x}: \mathcal{N}_{x}\left(\varepsilon^{*}\right) \rightarrow M$ is an isometry for any point $x \in K$. Call $N_{x}=\exp _{x}\left(\mathcal{N}_{x}\left(\varepsilon^{*}\right)\right)$. Moreover, for any $x \in K$ there is $\eta_{x}>0$ such that $X^{t}\left(N_{x}\right) \cap N_{x}=\emptyset$, for $t \in\left(-\eta_{x}, \eta_{x}\right)$, since there are no singularities in $\operatorname{cl}(K)$; and hence the function $x \mapsto \eta_{x}$ has a lower bound, say $\eta_{*}>0$. Define for any $x \in U_{1}$ and $s \in \mathbb{R}$ the map

$$
\tau_{x}^{s}: D_{x}^{s} \subset N_{x} \rightarrow \mathbb{R}
$$

using the Implicit Function Theorem, in such a way that $\tau_{x}^{s}(x)=s$ and $X^{\tau_{x}^{s}(y)}(y) \in N_{x_{s}}$, where $x_{s}=X^{s}(x)$. Whenever we speak about $D_{x}^{s}$, we are considering it as the maximal domain of definition. In spite of this, set

$$
R_{x}^{s}: D_{x}^{s} \rightarrow N_{x_{s}}
$$

such that $R_{x}^{s}(y):=X^{\tau_{x}^{s}(y)}(y), \forall y \in D_{x}^{s}$. Notice $D_{x} R_{x}^{s}=L_{x}^{s}$. Moreover, there exist a neighborhood $V_{s} \subset \mathcal{N}$ of the zero-section, where we can define a transformation $R^{s}: V_{s} \rightarrow N$ where

$$
V_{s} \ni(x, v) \stackrel{R^{s}}{\longmapsto}\left(X^{s}(x), \exp _{x_{s}}^{-1}\left(R_{x}^{s}\left(\exp _{x}(v)\right)\right)\right)
$$


This transformation covers the flow on $M$, i.e. the following diagram commutes:

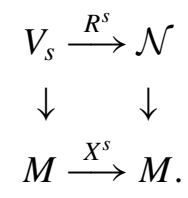

In fact, $\left[V_{s}\right]_{x} \supset \exp _{x}^{-1}\left(D_{x}^{s}\right)$, for all $x \in U_{1}$. Observe $\left\{R^{t}\right\}$ is a local flow on $\bigcap_{t \in \mathbb{R}} V_{t}$.

\section{Central manifolds}

Throughout this section we shall construct central-stable and central-unstable manifolds for invariant sets with dominated splitting. Moreover, we shall see they are of class $C^{2}$. Also we shall prove an analogous version of Pliss' Lemma (see [18]) for continuous-time dynamical systems. As a consequence of these, we shall obtain that there are only a finite number of irrational tori contained in $\Lambda$. At the end, we shall reduce the proof of Theorem B to a Main Lemma. Let $X \in \mathcal{X}^{2}(M)$ and $\Lambda \subset M$ be a compact invariant set with dominated splitting such that $\operatorname{Sing}(X) \cap \Lambda=\emptyset$. Also assume that all periodic orbits in $\Lambda$ are hyperbolic saddles. We begin establishing the following inequalities.

LEMMA 3.1. - If $\Lambda$ is an invariant set as above, there exist constants $C>0$ and $\sigma \in(0,1)$ such that for every $x_{0} \in \Lambda$ and for all $t \geqslant 0$ the following holds:

(1) $\left.\left.\left|L^{t}\right| E\left(x_{0}\right)|| L^{-t}\right|_{F\left(x_{t}\right)}\right|^{2} \leqslant C \sigma^{t}$,

(2) $\left.\left|L^{t}\right| E\left(x_{0}\right)\right|^{2}\left|L^{-t}\right|_{F\left(x_{t}\right)} \mid \leqslant C \sigma^{t}$.

Proof. - We only care about the first inequality since the other is analogous. Take $\alpha \in(0,1)$. We claim there is $T>0$ such that for any $x \in \Lambda$

$$
\frac{\left|L^{T}\right|_{E(x)} \mid}{\left.\left|L^{T}\right|_{F(x)}\right|^{2}} \leqslant \alpha .
$$

After the claim, it is not difficult to see how the lemma follows; we only have to notice that $\left.\left.\left|L^{t}\right|_{E}\left|=\prod_{j=1}^{\alpha}\right| L^{j T}\right|_{E}|| L^{r}\right|_{E} \mid$ where $t=\alpha T+r$, and the same for the subspace $F$. Hence, setting

$$
C=\max \left\{\left.\left|L^{r}\right|_{E(x)}|,| L^{r}\right|_{F(x)} \mid ; x \in \Lambda, r \in[0, T]\right\},
$$

we conclude

$$
\frac{\left|L^{t}\right|_{E\left(x_{0}\right)} \mid}{\left.\left|L^{t}\right|_{F\left(x_{t}\right)}\right|^{2}} \leqslant C\left(\alpha^{1 / t_{0}}\right)^{t} .
$$

However, if the claim do not happen then for all $T>0$ there is $x^{T} \in \Lambda$ such that

$$
\frac{\left|L^{T}\right|_{E\left(x^{T}\right)} \mid}{\left.\left|L^{T}\right|_{F\left(x^{T}\right)}\right|^{2}} \geqslant \alpha .
$$


Take the measures $\mu_{T}=\frac{1}{T} \int_{0}^{T} \delta_{x_{s}^{T}} \mathrm{~d} s$, where $x_{s}^{T}=X^{s}\left(x^{T}\right)$ and consider a subsequence $\mu_{i}:=\mu_{T_{i}}$ converging to a measure $\mu$, with $\operatorname{supp}(\mu) \subset \Lambda$. Observe the function $\frac{\left|L^{t}\right| E(x) \mid}{\left.\left|L^{t}\right| F\left(x_{t}\right)\right|^{2}}$ is $C^{1}$ with respect to $t$. Let

$$
\varphi(x)=\left.\frac{\mathrm{d}}{\mathrm{d} t} \log \left(\left.\frac{\left|L^{t}\right| E(x) \mid}{\left|L^{t}\right| F\left(x_{t}\right)}\right|^{2}\right)\right|_{t=0}
$$

and notice it is continous on $x$. It is true that $\int \varphi \mathrm{d} \mu \geqslant 0$. In fact,

$$
\int \varphi \mathrm{d} \mu=\lim _{i \rightarrow \infty} \varphi \mathrm{d} \mu_{i}=\lim \frac{1}{T_{i}} \int_{0}^{T_{i}} \varphi\left(x_{s}^{T_{i}}\right) \mathrm{d} s=\lim _{i \rightarrow \infty} \frac{1}{T_{i}} \log \left(\frac{\left|L^{t}\right| E(x) \mid}{\left.\left|L^{t}\right| F\left(x_{t}\right)\right|^{2}}\right) .
$$

Hence, the Theorem of Descomposition on Ergodic Measures implies there is an ergodic measure $v$ with $\operatorname{supp}(v) \subset \operatorname{supp}(\mu)$ and such that $\int \varphi \mathrm{d} v \geqslant 0$. Denote by $\lambda_{E}=$ $\lambda_{E}(v)=\int \log \left|L^{1}\right|_{E} \mid \mathrm{d} v$, and $\lambda_{F}=\lambda_{F}(v)=\int \log \left|L^{1}\right|_{F} \mid \mathrm{d} v$, the Lyapunov Exponents of $v$ with respect to $E$ and $F$, respectively. It is true that $\lambda_{E}<0$ and $\lambda_{F}<0$, since $\int \varphi \mathrm{d} v=\lambda_{E}-2 \lambda_{F} \geqslant 0$ and the condition of domination implies $\lambda_{E}<\lambda_{F}$. Hence $0 \leqslant \lambda_{E}-2 \lambda_{F}<\lambda_{F}-2 \lambda_{F}=-\lambda_{F}$. Therefore $\lambda_{E}<0$ and so $\lambda_{F}<0$. Now we claim that the support of $v$ is contained in the orbit of some periodic point of the flow, that must be a sink. Oseledets' Theorem [13], asserts that $v$-almost everywhere there is a unique invariant splitting $E_{1} \oplus[X] \oplus E_{2}$ for $X^{1}$. For that and since the splitting $E \oplus F$ is invariant by $L^{1}$ we can call $E_{i}$ that $E \oplus[X]=E_{1} \oplus[X]$ and $F \oplus[X]=E_{2} \oplus[X]$. Denote $\lambda_{1}, \lambda_{2}, \lambda_{[X]}=0$ their respective Lyapunov Exponents. Notice

$$
\begin{aligned}
\lambda_{E} & =\int \log \left(\left.L^{t}\right|_{E}\right) \mathrm{d} v=\int \log \left(\operatorname{det}\left(\left.D X^{1}\right|_{E \oplus[X]}\right)\right) \mathrm{d} v \\
& =\int \log \left(\operatorname{det}\left(\left.D X^{1}\right|_{E_{1} \oplus[X]}\right)\right) \mathrm{d} v=\lambda_{1}+\lambda_{[X]}=\lambda_{1} .
\end{aligned}
$$

Analogously $\lambda_{F}=\lambda_{2}$; therefore $\lambda_{1}>0$ and $\lambda_{2}>0$, and the claim follows.

Now, our setting fits into the arguments of [6] to prove the existence of central stable and central unstable manifolds tangent to directions $E$ and $F$, respectively. This is summarized in the following lemma.

LEMMA 3.2. - For each $\varepsilon>0$ denote the interval $I_{\varepsilon}=(-\varepsilon, \varepsilon)$ and let $\operatorname{Emb}^{2}\left(I_{1}, M\right)$ be the set of $C^{2}$ embeddings of $I_{1}$ on $M$. There exist two continous sections

$$
\Phi^{c s(c u)}: \Lambda \rightarrow \operatorname{Emb}^{2}\left(I_{1}, \mathcal{N}\right)
$$

such that, if we denote $W_{\varepsilon}^{c s(c u)}(x)=\Phi^{c s(c u)}(x)\left(I_{\varepsilon}\right)$, respectively, they verify the following properties:

(a) $T_{x} W_{\varepsilon}^{c s}(x)=E(x)$ and $T_{x} W_{\varepsilon}^{c u}(x)=F(x)$.

(b) For all $\varepsilon_{1} \in(0,1)$ there exists $\varepsilon_{2}>0$ such that $R^{t}\left(W_{\varepsilon_{2}}^{c s}(x)\right) \subset W_{\varepsilon_{1}}^{c s}\left(x_{t}\right)$ and $R^{-t}\left(W_{\varepsilon_{2}}^{c u}(x)\right) \subset W_{\varepsilon_{1}}^{c u}\left(x_{-t}\right)$, for all $t \in[0,1]$. 
Notice that $\Phi^{c s}(x)\left(I_{1}\right) \subset \mathcal{N}_{x}$ since $\Phi^{c s}$ is a section on $\mathcal{N}$ and the same for $\Phi^{c u}$. We shall call the manifold $W_{(\varepsilon)}^{c s}$ the (local) central stable manifold and $W_{(\varepsilon)}^{c u}$ the (local) central unstable manifold. Observe that the second part of the lemma implies the next corollary:

COROLlary 3.3. - Given $\epsilon>0$ there is $\delta>0$ such that:

(a) If $y \in W_{\varepsilon}^{c s}(x)$ and $\operatorname{dist}\left(x_{t}, y_{t}\right) \leqslant \delta$ for all $t \in\left(0, t_{0}\right)$, then $R^{t}(y) \in W_{\varepsilon}^{c s}\left(x_{t}\right)$ for $t \in\left(0, t_{0}\right)$.

(b) If $y \in W_{\varepsilon}^{c u}(x)$ and $\operatorname{dist}\left(x_{-t}, y_{-t}\right) \leqslant \delta$ for all $t \in\left(0, t_{0}\right)$, then $R^{-t}(y) \in W_{\varepsilon}^{c s}\left(x_{-t}\right)$ for $t \in\left(0, t_{0}\right)$.

It is not difficult to see that in our context it is true the following

COROLlARY 3.4. - Let $x \in \Lambda$ such that for some $\gamma \in(0,1)$ we have

$$
\left|L^{t}\right|_{E(x)} \mid \leqslant \gamma^{t}, \quad \text { for all } t \geqslant 0 .
$$

Then there exists $\varepsilon>0$ such that $\left|R^{t}\left(W_{\varepsilon}^{c s}(x)\right)\right| \rightarrow 0$ as $t \rightarrow \infty$; that means the central stable manifold is in fact a stable one.

\subsection{Hyperbolic times of a point}

It is possible to prove a version of Pliss' Lemma [18] for flows. In this case, the proof is easier than in the case of diffeomorphisms; see [8].

THEOREM 3.5. - Given any $\varepsilon>0, A \in \mathbb{R}$ and $c>0$, there exists $K>0$ such that if $H:[0, T] \rightarrow \mathbb{R}$ is diferentiable, $H(0)=0, H(T)<c T$ and $\inf \left(H^{\prime}\right)>A$, then the set

$$
\mathcal{P}_{\varepsilon}=\{\tau \in[0, T] \mid H(s)-H(\tau) \leqslant(c+\varepsilon)(s-\tau) \text { for all } s \in[\tau, T]\}
$$

has Lebesgue measure greater than $T K$.

Proof. - Take $\varepsilon>0$ and $A$ as in the hypothesis. Define $G(s)=H(s)-(c+\varepsilon) s$. We assume $G$ is of class $C^{2}$ and it does not have degenerate critical points; that is, $G^{\prime}(x)=0$ if and only if $G^{\prime \prime}(x) \neq 0$. Actually we can assume all their critical values are different, otherwise, an approximation of $G$ still works. Since $G(0)=0$ and $G(T)<-\varepsilon T$, it is possible to define two (perhaps finite) sequences, say $\left\{a_{i}\right\}$ consisting of critical points of $G$ such that $G(x)<G\left(a_{i}\right)$ for every $x>a_{i}$ (if finite, set the last point $a_{n+1}=T$, if it is not so, then $\left.a_{i} \rightarrow T\right)$ and $\left\{b_{i}\right\}$ as the first number $b>a_{i}$ such that $G\left(b_{i}\right)=G\left(a_{i+1}\right)$. Of course $b_{i} \leqslant a_{i+1}$. Let $B=-\inf G^{\prime}$, then by the Mean Value Theorem

$$
\frac{G\left(a_{i}\right)-G\left(b_{i}\right)}{B} \leqslant b_{i}-a_{i} .
$$

On the other hand, the union $\bigcup_{i}\left(a_{i}, b_{i}\right)$ is contained in $B_{\varepsilon}$. In fact,

$$
\begin{aligned}
H(s)-H(\tau) & =H(s)-(c+\varepsilon) s-(H(\tau)-(c+\varepsilon) \tau)+(c+\varepsilon)(s-\tau) \\
& =G(s)-G(\tau)+(c+\varepsilon)(s-\tau) .
\end{aligned}
$$

Hence, $G(s)-G(\tau)+(c+\varepsilon)(s-\tau)<(c+\varepsilon)(s-\tau)$ if and only if $G(s)<G(\tau)$. Let $t \in\left(a_{i}, b_{i}\right)$, for some $i$. If for a given $s>\tau$ we have $G(s)=G(\tau)$ then $\tau=b_{i}$, because $b_{i}$ is the first number $b>a_{i}$ such that $G(b)=G\left(a_{i+1}\right)$; a contradiction. Therefore, 


$$
\begin{aligned}
\operatorname{leb}\left(\mathcal{P}_{\varepsilon}\right) & \geqslant \sum_{i=1}^{n}\left(b_{i}-a_{i}\right) \geqslant \frac{1}{B} \sum_{i=1}^{n} G\left[\left(a_{i}\right)-G\left(b_{i}\right)\right] \\
& =\frac{1}{B} \sum_{i=1}^{n}\left[G\left(a_{i}\right)-G\left(a_{i+1}\right)\right]=\frac{1}{B}\left(G\left(a_{1}\right)-G(T)\right) .
\end{aligned}
$$

Notice $G\left(a_{1}\right) \geqslant 0$ since it is a global maximum. Therefore $\operatorname{leb}\left(\mathcal{P}_{\varepsilon}\right) \geqslant \frac{-G(T)}{B} \geqslant \frac{\varepsilon T}{B}$, and then $\operatorname{leb}\left(\mathcal{P}_{\varepsilon}\right) T^{-1} \geqslant \varepsilon B^{-1}$. Finally $G^{\prime}(t)=H^{\prime}(t)-(c+\varepsilon)$ and $B=\inf \left(G^{\prime}\right)=$ $(c+\varepsilon)-A \geqslant c+\varepsilon-A$, that is

$$
\frac{\operatorname{leb}\left(\mathcal{P}_{\varepsilon}\right)}{T} \geqslant \frac{\varepsilon}{c+\varepsilon-A}=: K
$$

COROLlary 3.6. - Let $X \in \mathcal{X}^{1}(M)$ and let $\Lambda$ be an invariant compact set with dominated splitting. Given $\varepsilon>0, \gamma<1$ and $x \in \Lambda$. If there is $T>0$ such that $\left|L^{T}\right| E(x) \mid<\gamma^{T}$, then there is $K>0$ such that the set

$$
\mathcal{P}_{\varepsilon}(x)=\left\{\tau \in[0, T]|| L^{s-\tau}|E(x)| \leqslant\left(\mathrm{e}^{\log (\gamma)+\varepsilon}\right)^{s-\tau} \forall s \in[\tau, T]\right\}
$$

is such that $\operatorname{leb}\left(P_{\varepsilon}(x)\right) \geqslant K T$, and hence non-empty.

Proof. - Recall the function $H_{E}(t)=\log \left(\left|L^{t}\right|_{E(x)} \mid\right)$ is $C^{1}$. Moreover $\mathrm{d} / \mathrm{d} t\left(H_{E}\right)=$ $\left\langle D_{x_{t}} X(E), E\right\rangle$, and hence $\left|H^{\prime}(t)\right| \leqslant\|X\|_{C^{1}}$. Also remember

$$
D R_{x}^{t}(v)=D_{x} X^{t}(v)-\frac{\left\langle D_{x} X^{t}(v), X(x)\right\rangle}{\|X(x)\|^{2}} X(x) .
$$

COROLlaRY 3.7. - Given $\varepsilon>0, \gamma<1$ and $x_{0} \in \Lambda$ if there exists $t>0$ such that $\left|L^{s}\right|_{E\left(x_{t}\right)} \mid<\gamma^{s}$, for all $s \geqslant 0$, then for $\tilde{\lambda}=\mathrm{e}^{\log (\gamma)+\varepsilon}$, the set

$$
\mathcal{P}\left(x_{0}, \tilde{\lambda}\right)=\left\{\left.\tau \in \mathbb{R}|| L^{s}\right|_{E\left(x_{\tau}\right)} \mid \leqslant \tilde{\lambda}^{s} \forall s>0\right\}
$$

is unbounded.

We call the set on (2) the hyperbolic times of $x_{0}$ related to $\tilde{\lambda}$.

\subsection{Reducing Theorem $B$ to a Main Lemma}

Now, it is not difficult to see that Theorem B follows from the Theorem 3.8 below, once we guarantee there can only be finitely many irrational tori on $\Lambda$.

THEOREM 3.8. - Let $X \in \mathcal{X}^{2}(M)$ and let $\Lambda$ be a compact invariant for $X$ set with dominated splitting; also assume all periodic points in $\Lambda$ are hyperbolic of saddle type and $\operatorname{Sing}(X) \cap \Lambda=\emptyset$. Then one of the following happens:

(1) $\Lambda$ is an hyperbolic set.

(2) There exist an irrational torus $\mathcal{T} \subset \Lambda$.

PROPOSITION 3.9. - There are only finitely many irrational tori on $\Lambda$. 
Proof. - Assume by contradiction there are infinitely many irrational tori, named $\left\{\mathcal{I}_{k}\right\}_{k \in \mathbb{N}}$. Take numbers $\lambda<\gamma_{1}<\gamma_{2}<1$ and $c>0$ such that $(1+c) \lambda<1$. On each $\mathcal{T}_{k}$ there exist a point $x^{k}$, and some $t_{k}>0$ such that

$$
\left|L_{F\left(x^{k}\right)}^{t}\right| \leqslant(1+c)^{t}, \quad \forall t \geqslant t_{k}
$$

and then, by the domination condition we have

$$
\left|L_{E\left(x^{k}\right)}^{t}\right| \leqslant \lambda^{t}(1+c)^{t}<\gamma_{1}^{t}, \quad \forall t \geqslant t_{k} .
$$

An application of Theorem 3.5 imply there exist $T_{k}>0$ such that for $y^{k}=X^{T_{k}}\left(x^{k}\right)$, $\left|L^{t}\right|_{E\left(y^{k}\right)} \mid \leqslant \gamma_{2}^{t}$, for all $t>0$. Hence, Corollary 3.4 let us conclude that each $y^{k}$ has a stable manifold of size $\varepsilon>0$.

Let $y \in \Lambda$ be an accumulation point of the $\left\{y^{k}\right\}$ and denote by $\Sigma_{y} \subset N_{y} \subset M$ an open ball around $y$ of radius smaller than $\varepsilon / 3$. Still denote $y^{k}$ the points on $\Sigma_{y}$ which are immediate iterates of the true $y^{k}$. Actually, they also have stable manifolds of uniform size $\varepsilon$. Notice that it is not possible $\mathcal{T}_{k} \cap \Sigma_{y}$ be a circle for infinitely many $k$, because the splitting on $\Lambda$ is continuous. Moreover, $\mathcal{T}_{k} \cap \Sigma_{y}$ is an arc that meets the boundary of the ball in two different connected components. So, it is not difficult to see there are $\mathcal{T}_{j}$ and $y^{k} \in \mathcal{T}_{k} \cap \Sigma_{y}, j \neq k$, that $\mathcal{T}_{j} \cap \Sigma_{y} \neq \emptyset$ and $W_{\varepsilon}^{s}\left(y^{k}\right) \cap \mathcal{T}_{j} \neq \emptyset$. This contradiction proves the proposition.

In [21], they prove Theorem 3.8 is reduced to the next Main Lemma, and their arguments work for both, diffeomorphisms and vector fields. We only notice that in spite of Lemma 2.2, it is elementary to verify that if $\Lambda$ is a compact invariant set with dominated splitting such that for every $x \in \Lambda$ we have $\left|L^{t}\right|_{E(x)} \mid \rightarrow 0$ and $\left|L^{-t}\right|_{F(x)} \mid \rightarrow 0$ as $t \rightarrow \infty$, then $\Lambda$ is a hyperbolic set.

MAIN LEMma 1. - Let $\Lambda_{0} \subset M$ be a compact transitive invariant set for $X^{t}$ with a dominated splitting such that $\Lambda_{0} \cap \operatorname{Sing}(X)=\emptyset$ and it is not an irrational torus neither a periodic orbit. If every properly contained invariant subset of $\Lambda_{0}$ is hyperbolic, then $\Lambda_{0}$ is hyperbolic itself.

\section{Asymptotical behavior of dynamical intervals}

In order to obtain hyperbolicity over an invariant set with dominated splitting, one must show that the central manifolds on each point are actual stable and unstable manifolds. This is the aim of this section. For that, we shall prove a Denjoy's like property which roughly speaking states that smoothness on the vector field $X$ do not allow the existence of some kind of wandering intervals. (The precise statement is Proposition 4.2.) Let $X \in \mathcal{X}^{2}(M)$ and let $\Lambda$ be compact invariant set with dominated splitting such that $\operatorname{Sing}(X) \cap \Lambda=\emptyset$ and all periodic points in $\Lambda$ are hyperbolic saddles. Consider the set

$$
\Lambda^{+}:=\bigcap_{t \geqslant 0} X^{-t}(\operatorname{cl}(V))
$$


for some neighborhood $V$ such that $\bar{V} \subset U_{1}$. Recall $U_{1}$ is a neighborhood of $\Lambda$ where it is possible to define an extension of the invariant 1-cone field on $\Lambda$ (see Section 2.1). Notice that this induces an extension of the dominated splitting of $\Lambda$ over $\Lambda^{+}$.

We say $I \subset \mathcal{N}_{x}$ or $I \subset N_{x}$ are intervals if they are the image of a smooth function $\varphi:[0,1] \rightarrow \mathcal{N}_{x}$ or $N_{x}$, respectively. Denote by $|I|$ the length of the interval $I$.

The following definitions collect the insight of central unstable manifolds and the property that will make them actual unstable manifolds.

DEFINITION 2. - Let I be an interval contained in $\mathcal{N}_{x_{0}}$ for some $x_{0} \in I$ and let $\delta>0$.

(1) We say $I$ is a-transversal to the $E$ direction for some a $\in(0,1]$ if $T_{y} I \subset C_{a}^{F}(y) \subset$ $\mathcal{N}_{x_{0}}$ for any $y \in I$. It is $E$-transversal if it is a-transversal to the direction $E$ for some $0<a \leqslant 1$.

(2) We say $I$ is a $(\delta, E)$-interval if it is E-transversal, $I \subset \bigcap_{t \geqslant 0} V_{t}$ and $\left|R^{t}(I)\right| \leqslant \delta$ for all $t \geqslant 0$.

Although these definitions are stated for intervals contained in the normal bundle, we say an interval $I \subset N_{x}$ on $M$ is $E$-transversal or $(\delta, E)$-transversal if the interval defined by $\hat{I}:=\exp _{x}^{-1}(I)$ is. Notice if $\hat{I} \subset \mathcal{N}$ is a $(\delta, E)$-interval, then $\exp _{x}(\hat{I}) \subset \Lambda^{+}$.

Remark 1. - For any $c>0$ there is $\delta_{c}>0$ such that if $I$ is a $(\delta, E)$-interval associated to $x \in I$ with $\delta \leqslant \delta_{c}$ then, for any $y \in I$ :

$$
(1-c)^{t} \leqslant \frac{\left|D_{y} R_{x}^{t}\right|_{E(y)} \mid}{\left|L^{t}\right|_{E(x)} \mid} \leqslant(1+c)^{t} .
$$

Also is true a similar statement with respect to the $F$-direction.

For $(\delta, E)$-intervals contained in sets with dominated splitting we have stable manifolds, as we shall see on the next lemma. An $E$-transversal interval which do not grow its length on future iterations, it must contract exponentially along the $E$-direction.

LEMMA 4.1. - For any $\gamma \in(\lambda, 1)$ there is $\delta_{1}>0$ such that if $I$ is a $(\delta, E)$-interval, $I \subset \mathcal{N}_{x}, x \in I$, with $\delta \leqslant \delta_{1}$, then there is $t_{0}>0$ such that

$$
\left|L^{s}\right|_{E\left(x_{t_{0}}\right)} \mid<\gamma^{s}, \quad \forall s \geqslant 0 .
$$

Moreover, for any $y \in \hat{I}_{t_{0}}=\exp _{x_{t_{0}}}\left(R^{t_{0}}(I)\right)$ we have

$$
\left|D_{y} R^{s}\right|_{E\left(x_{t_{0}}\right)} \mid<\gamma^{s}, \quad \forall s \geqslant 0
$$

and hence, on any point of $\hat{I}_{t_{0}}$ we have a stable manifold of uniform size.

Proof. - Fix $\gamma \in(\lambda, 1)$ and $\lambda_{2}<1$ such that $\lambda \lambda_{2}^{-1}<\gamma$. Let $c>0$ and $\lambda_{3}<1$ such that $(1-c) \lambda_{2}<\lambda_{3}$. Take $\delta_{1} \leqslant \delta_{c}$ of Remark 1 , and let $I$ be a $(\delta, E)$-interval with $\delta \leqslant \delta_{1}$, associated to $x_{0} \in I$.

We claim there is $t_{0}>0$ such that $\left|L^{s}\right|_{F\left(x_{t_{0}}\right)} \mid<\lambda_{2}^{-s}$, for all $s>0$. To prove the claim suppose there exist a sequence $s_{t} \rightarrow \infty$ as $t \rightarrow \infty$ such that for all $t \geqslant 0$

$$
\left|L^{s_{t}}\right|_{F\left(x_{t}\right)} \mid \geqslant \lambda_{2}^{-s_{t}}
$$


Since $\left|\hat{I}_{t}\right| \leqslant \delta_{c}$, we have:

$$
(1-c)^{-s} \leqslant \frac{\left|D_{y} R_{x_{t}}^{s}\right|_{\tilde{F}(y)} \mid}{\left|L^{s}\right|_{F\left(x_{t}\right)} \mid} \leqslant(1+c)^{s} .
$$

Then

$$
\left.\left|D_{y} R_{x_{t}}^{s_{t}}\right|_{\tilde{F}(y)}\left|\geqslant(1-c)^{-s_{t}}\right| L^{s_{t}}\right|_{F\left(x_{t}\right)} \mid \geqslant\left((1-c) \lambda_{2}\right)^{-s_{t}} \geqslant \lambda_{3}^{-s_{t}} .
$$

It is a straight forward computation that then, $\left|I_{t+s_{t}}\right| \geqslant \lambda_{3}^{-s_{t}}\left|I_{t}\right|$. If $t$ is big enough, we achieve a contradiction to $\left|I_{t+s_{t}}\right| \leqslant \delta_{c}$. Hence, the claim follows.

Actually, we have proved that $\left|L^{s}\right|_{E\left(x_{t_{0}}\right)} \mid \leqslant\left(\lambda \lambda_{2}^{-1}\right)^{s}$, for all $s \geqslant 0$. Notice that if $c>0$ is such that $(1+c) \lambda \lambda_{2}^{-1}<\gamma$ and $\delta>0$ is small enough that for any $y \in \hat{I}_{t_{0}}$ and $s \geqslant 0$ we have

$$
(1-c)^{-s} \leqslant \frac{\left|D_{y} R_{x_{t}}^{s}\right|_{E(y)} \mid}{\left|L^{s}\right|_{E\left(x_{t}\right)} \mid} \leqslant(1+c)^{s},
$$

then $\left|D_{y} R_{x_{t}}^{s}\right|_{E(y)} \mid<\gamma^{s}$, for all $y \in \hat{I}_{t_{0}}$ and for all $s \geqslant 0$, as we required. Corollary 3.4 guarantees then stable manifolds on points of $\hat{I}_{t_{0}}$ of uniform size.

Maximal $(\boldsymbol{\delta}, \boldsymbol{E})$-intervals. Let $I \subset \mathcal{N}_{x_{0}}$ be a $(\delta, E)$-interval associated to some $x_{0} \in I$. Denote by $I_{t}:=R^{t}(I)$ for $t \in \mathbb{R}^{+}$. We seek for a family of $(\delta, E)$-intervals $J_{t}$ such that $I_{t} \subset J_{t}$ and also $R^{t-s}\left(J_{s}\right) \subset J_{t}$ for all $0<s<t$, and any $t \in \mathbb{R}^{+}$; being maximal for these properties. In order to find it we appeal to Zorn's Lemma in the following way: Consider the set of functions from $\mathbb{R}$ into the compact connected subsets of the normal bundle $\mathcal{N}$, denoted by $P(\mathcal{N})$ :

$$
\begin{aligned}
\mathcal{F}=\{J: \mathbb{R} \rightarrow P(\mathcal{N}) \mid & J(s) \subset \mathcal{N}_{x_{s}}, I_{s} \subset J(s), J(s) \text { is a }(\delta, E) \text {-interval } \\
& \text { and } \left.R^{t}(J(s)) \subset J(s+t), \forall s, t \in \mathbb{R}^{+}\right\}
\end{aligned}
$$

with the order relation: $J \leqslant K$ if and only if $J(s) \subset K(s), \forall s \in \mathbb{R}^{+}$. Observe that the function $I(\cdot)=R^{(\cdot)}(I)$ belongs to $\mathcal{F}$. Moreover, any totally ordered chain has a supremum in $\mathcal{F}$, say, $\left\{J^{i} \in \mathcal{F} ; i \in \Gamma\right\}$, then $J^{\infty}(s)=\bigcup_{i \in \Gamma} J^{i}(s)$, defined for each $s \in \mathbb{R}$ belongs to $\mathcal{F}$. Therefore, there is a maximal element on $\mathcal{F}$ denoted by $J_{s}:=J(s)$. If there is an interval $\tilde{K} \subset N_{x_{s}}$ such that $J_{s} \subset \tilde{K}$ for some $s \in \mathbb{R}^{+}$and $\left|R^{t}(\tilde{K})\right| \leqslant \delta$ for all $t \geqslant 0$, then the family $K(t):=J_{t}$ for $t<s$ and $K(t):=R^{t}(\tilde{K})$ for $t \geqslant s$ belongs to $\mathcal{F}$; contradicting the fact $J(\cdot)$ is a maximal element of $\mathcal{F}$.

Notice that Lemma 4.1 implies the existence of $\delta_{0}>0$ such that if $\left\{J_{S}\right\}$ is a maximal family of $(\delta, E)$-intervals related to a $(\delta, E)$-interval, say $I$, with $\delta \leqslant \delta_{0}$. Then, there exist numbers $s>0$ and $\varepsilon_{0}>0$ such that for every $y \in J_{s}$, the center-stable manifold $W_{\varepsilon_{0}}^{c s}(y)$ is in fact a true stable manifold for $R^{t}$, that is, $\left|R^{t}\left(W_{\varepsilon_{0}}^{c s}(y)\right)\right| \rightarrow 0$ as $t \rightarrow \infty$. Moreover there exist $C>0$ and $\gamma \in(0,1)$ such that

$$
\left|R_{x_{s}}^{t}\left(\exp _{x_{s}}\left(W_{\varepsilon_{0}}^{s}(y)\right)\right)\right|<C \gamma^{t} \quad \text { for any } t \in \mathbb{R},
$$

that is, $\exp _{x_{s}}\left(W_{\varepsilon_{0}}^{s}(y)\right)$ is a local stable manifold for $R_{x_{s}}^{t}$. We will denote also $W_{\varepsilon_{0}}^{s}(y):=$ $\exp _{x_{s}}\left(\hat{W}_{\varepsilon_{0}}^{s}(y)\right)$, except when it is relevant to remark the difference. For any $s \in \mathcal{P}$ denote by $J_{s}^{\varepsilon}:=\bigcup_{y \in J_{s}} W_{\varepsilon}^{s}(y) \subset M$. 


\subsection{Denjoy's like property}

Let $\hat{I} \subset \mathcal{N}_{x_{0}}$ for some $x_{0} \in \hat{I}$ be a $(\delta, E)$-interval. Denote by $I_{t}=\exp _{x_{0}}\left(\hat{I}_{t}\right) \subset M$, where $\hat{I}_{t}=R^{t}(\hat{I})$ and $x_{t}:=X^{t}(x)$. Define the $\omega$-limit set of $I_{0}$ as $\omega\left(I_{0}\right)=\bigcup_{y \in I_{0}} \omega(y)$; where $\omega(y)$ is the usual $\omega$-limit set of $y$ according to the flow $X^{t}$.

Proposition 4.2. - There is $\delta_{0}>0$ such that if $I$ is a $(\delta, E)$-interval associated to some $x \in I$ for some $\delta<\delta_{0}$, then there are two possibilities: $\omega(I) \subset \operatorname{Per}\left(X^{t}\right)$ or $\omega(I)=\mathcal{T}$, where $\mathcal{T}$ is an irrational torus.

Proof. - Let $\lambda<1$ be the number in the dominated splitting condition. Fix $\gamma \in(\lambda, 1)$ and take $\delta_{1}>0$ from Lemma 4.1. Let $I_{0} \subset M$ be a $(\delta, E)$-interval associated to $x_{0}$ with $\delta \leqslant \delta_{1}$. Denote by $I_{t}=R_{x}^{t}\left(I_{0}\right)$. Let $\left\{\hat{J}_{s} \mid s \in \mathbb{R}^{+}\right\}$be a maximal family of $(\delta, E)$-intervals for $\hat{I}_{s}=\exp _{x_{s}}^{-1}\left(I_{s}\right)$ constructed above and denote $J_{s}=\exp _{x_{s}}\left(\hat{J}_{s}\right) \subset M$. Lemma 4.1 implies that there exist $t_{0}>0$ for which we have defined a stable manifold of size $\varepsilon_{0}$ on any point of $J_{t_{0}}$. This fact together with Corollary 3.7 imply that the set of hyperbolic times of $x_{0}$ related to $\gamma, \mathcal{P}\left(x_{t_{0}}\right)$, is unbounded. Corollary 3.4 assert that for any $s \in \mathcal{P}\left(x_{t_{0}}\right)$ and on any point of $J_{s}$, we also have stable manifolds of size $\varepsilon_{0}$. Since we are interested on asymptotical properties of $I$ we shall assume $t_{0}=0$; in fact, $w(I) \subset w\left(J_{t_{0}}\right)$. We shall denote, by simplicity $\mathcal{P}=\mathcal{P}\left(x_{0}\right)$. The following situations requires two different arguments:

(I) There exist some $t, s \in \mathcal{P}, t<s$, for which $J_{s}^{\varepsilon} \cap\left[R^{t-s}\left(J_{s}\right)\right]^{\varepsilon} \neq \emptyset$.

(II) For any $t, s \in \mathcal{P}$, we have $J_{s}^{\varepsilon} \cap\left[R^{t-s}\left(J_{s}\right)\right]^{\varepsilon}=\emptyset$.

Let us first deal with case I. For that, we shall assume first that $\lim _{s \in \mathcal{P}}\left|\hat{J}_{S}\right|=0$. If we can guarantee the hypothesis of the following lemma we shall conclude the thesis of Proposition 4.2 in this case.

LemmA 4.3. - If there exist $s \in \mathcal{P}$ and $t>0$ such that $R_{x_{s}}^{t}\left(J_{s}\right) \subset J_{s}^{\varepsilon}$, then there is a periodic orbit $p \in \Lambda$ such that $\omega(I) \subset \omega\left(J_{0}\right)=\mathcal{O}(p)$.

Proof. - Define $\pi: J_{s}^{\varepsilon} \rightarrow J_{s}$, a projection along stable manifolds. Then $\pi \circ R_{x_{s}}^{t}: J_{s} \rightarrow$ $J_{s}$ is a continuous map of the interval. Since $R_{x_{s}}^{t}\left(J_{s}\right) \subset J_{s}^{\varepsilon}$, we see $\pi\left(R_{x_{s}}^{t}\left(J_{s}\right)\right) \subset J_{s}$. Therefore, there is $z \in J_{s}$ such that $R_{x_{s}}^{t}(z) \in W_{\varepsilon}^{s}(z)$; and this can only happen if both $z, R_{x_{s}}^{t}(z) \in W^{s}(p)$ of some periodic point $p \in \Lambda$. Moreover, $p$ must be a sink and $\omega(I) \subset \omega\left(J_{0}\right)=\mathcal{O}(p)$.

The following two claims let us fit into the hypothesis of the previous lemma.

Claim 1. - There is $\delta^{\prime}>0, s_{0}>0$ and $c>0$ such that, if $\tilde{J}$ is a $\left(\delta^{\prime}, E\right)$-interval satisfying $J_{s}^{c \varepsilon} \cap \tilde{J} \neq \emptyset$, with $s_{0}<s \in \mathcal{P}$, then $\tilde{J} \subset J_{s}^{\varepsilon}$.

Claim 2. - There is $s \in \mathcal{P}, s>s_{0}$ and $t>0$ in such a way $W_{c \varepsilon}\left(J_{s}\right) \cap R_{x_{s}}^{t}\left(J_{s}\right) \neq \emptyset$, and $R_{x_{s}}^{t}\left(J_{s}\right)$ is a $\left(\delta^{\prime}, E\right)$-interval.

Proof of Claim 1. - Let $\eta_{1}>0$ such that $\left(1+\eta_{1}\right) \lambda<1$. As we see in Remark 1, there is $\delta_{\eta_{1}}>0$ such that if $K$ is a $\left(\delta_{\eta_{1}}, E\right)$-interval we have $\forall z, z_{0} \in K$

$$
\frac{\left|D_{z} R^{t}\right|_{E(z)} \mid}{\left|D_{z_{0}} R^{t}\right|_{E\left(z_{0}\right)} \mid}<\left(1+\eta_{1}\right)^{t}, \quad \forall t \geqslant 0 .
$$


Let us suppose there is $\tilde{J}$ a $\left(\delta_{\eta_{1}}, E\right)$-interval such that $J_{s}^{c \varepsilon} \cap \tilde{J} \neq \emptyset$ for some $c>0$. Observe $\tilde{J} \subset D_{x_{s}}^{t}$, for all $t \geqslant 0$. Then there are points $z_{0} \in \tilde{J}$ and $y_{0} \in J_{s}$ such that $z_{0} \in W_{c \varepsilon}^{s}\left(y_{0}\right)$. Moreover, for any $c>0$ there is a positive $\eta_{c}>0$ such that

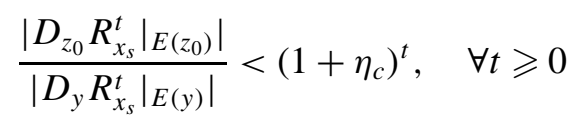

since both points lay on the same stable manifold $W_{c \varepsilon}^{s}(y)$.

Notice that if $\tilde{\lambda}_{c}=\left(1+\eta_{1}\right)\left(1+\eta_{c}\right) \lambda<1$, inequalities (3) and (4) imply that on any point of $\tilde{J}$ there are stable manifolds of size $\varepsilon^{\prime}\left(\tilde{\lambda}_{c}\right)>0$. Actually, $\eta_{c} \rightarrow 0$ as $c \rightarrow 0$ and $\varepsilon^{\prime}\left(\tilde{\lambda}_{c}\right)$ do not decreases. So, $\left|D_{z}\left(R_{x_{s}}^{t}\right)\right|_{E(z)} \mid<\tilde{\lambda}_{c}^{t}$ for $t \geqslant 0$ and all $z \in \tilde{J}$.

Fix $c \in\left(0, \frac{1}{2}\right)$ such that $\tilde{\lambda}_{c}<1$ and take $\tilde{\varepsilon}>0$ such that $c \varepsilon<\tilde{\varepsilon}<(1-c) \varepsilon$ and $\tilde{\varepsilon} \leqslant \varepsilon^{\prime}\left(\tilde{\lambda}_{c}\right)$. Take $s_{0} \in \mathcal{P}$ in such a way that for any $s>s_{0}$ we have $2\left|J_{s}\right|<\delta$. Then for all $z \in \tilde{J} \cap J_{s}^{c \varepsilon}$ we have $W_{\tilde{\varepsilon}}^{s}(z) \subset J_{s}^{\varepsilon}$ and $W_{\tilde{\varepsilon}}^{s}(z) \cap J_{s} \neq \emptyset$.

On the other hand, there is some constant $C>0$ such that for any $E$-transversal interval $A \subset \tilde{J}^{\tilde{\varepsilon}}$ we have $\left|R^{t}(A)\right| \leqslant C\left|R^{t}(\tilde{J})\right|$ for any $t \geqslant 0$. Take $\delta^{\prime}$ such that $\delta / 2+$ $C \delta^{\prime}<\delta$. If $\tilde{J}$ is not properly contained in $J_{s}^{\varepsilon}$, there is a $C^{2}$ extension of the interval $J_{s}$, named $J_{s} \cup A$ with $A \subset \tilde{J} \tilde{\varepsilon}$, which is $E$-transversal. Moreover,

$$
R^{t}\left(J_{s} \cup A\right) \leqslant\left|R_{x_{s}}^{t}\left(J_{s}\right)\right|+\left|R_{x_{s}}^{t}(A)\right| \leqslant \delta / 2+C \delta^{\prime}<\delta .
$$

The last inequality contradicts the fact of $J_{s}$ being maximal $(\delta, E)$-interval, and give us the claim.

Proof of Claim 2. - Actually, in the previous argument we could take $s_{0}$ big enough in such a way that $\left|J_{S}\right|<\delta^{\prime}$ and $\lambda^{s_{0}}<c$ also. The intersection property (hypothesis of case I) implies

$$
J_{s}^{c \varepsilon} \cap R_{x_{s}}^{t}\left(J_{s}\right) \neq \varnothing
$$

for some positive $t$. Since $R_{x_{s}}^{t}\left(J_{s}\right)$ is a $\left(\delta^{\prime}, E\right)$-interval we are done.

Now we assume $\lim \sup _{s \in \mathcal{P}}\left|J_{s}\right|>0$. Considering any convergent subsequence we obtain a limit interval $J_{s} \rightarrow J$ provided of a stable manifolds, say, $J^{\varepsilon}:=\bigcup_{z \in J} W_{\varepsilon}^{s}(z)$ for any $\varepsilon<\varepsilon_{0}$. There is no harm if we assume that $J^{\varepsilon_{0}}$ lies in some $N_{x}$ for some $x \in J \cap \Lambda$.

Lemma 4.4. - If $p \in J^{\varepsilon} \cap \operatorname{Per}\left(X^{t}\right)$ then $p$ is hyperbolic and $\omega(J) \subset \operatorname{Per}\left(X^{t}\right)$.

Proof. - Notice that $p \in \Lambda^{+}$. Let us suppose it is a hyperbolic saddle. Observe that $J \cap W_{\varepsilon}^{u}(p)$ is non-empty and transversal. Then, the Inclination Lemma ( $\lambda$-Lemma in [15]) implies that $\left|W_{\varepsilon}^{u}(p)\right| \leqslant \delta$, since $\left|R_{x}^{t}(J)\right| \leqslant \delta$ for all $t \geqslant 0$. Hence, there must be two periodical sinks $p_{1}, p_{2}$ bounding a cylinder that contains the orbit of $p$. Therefore, if $y \in J$ the $\omega$-limit of $y$ is either $p_{1}, p_{2}$ or $p$. A similar argument implies the thesis in the case of $p$ being a sink or a saddle node, and these are the only possibilities; since $p \in J^{\varepsilon}$ has a stable manifold.

Hence, we assume there are no periodic orbits passing through $J^{\varepsilon}$. Notice that the set $\partial J^{\varepsilon}$ consists of boundary of two kinds: $\partial^{s}\left(J^{\varepsilon}\right):=\bigcup_{z \in \partial J} W_{\varepsilon}^{s}(z)$ and $\partial^{u}\left(J^{\varepsilon}\right):=$ $\partial\left(J^{\varepsilon}\right) \backslash \partial^{s}\left(J^{\varepsilon}\right)$. This distinction allows us to make the next definition. 
DEFINITION 3. - If a set $A$ is such that $A \cap J^{\varepsilon} \neq \emptyset$ we say $A$ is stably contained on $J^{\varepsilon}$ if $A \cap \partial^{u}\left(J^{\varepsilon}\right)=\emptyset$, and we denote it by $A \subset^{s} J^{\varepsilon}$. We also define, for positive $\varepsilon$ and $\tilde{\varepsilon}$, the number $\rho\left(J^{\varepsilon}, J^{\tilde{\varepsilon}}\right)=\rho \in \mathbb{R}^{+}$as the first time such that $R_{x}^{\rho}\left(J^{\varepsilon}\right) \cap J^{\tilde{\varepsilon}} \neq \emptyset$.

Proposition 4.5. - There exists two subintervals $\tilde{L} \subset L \subset J$ such that $w(J)=$ $w(L)$, numbers $0<t_{0}<t_{1}$ and $\eta>0$ such that:

(1) $R_{x}^{t_{0}}\left(L^{\eta}\right) \subset^{s} L^{\eta}$ for $t_{0}=\rho\left(L^{\eta}, L^{\eta}\right)$, and $R_{x}^{t_{0}}\left(L^{\eta}\right) \cap \tilde{L}^{\eta}=\emptyset$.

(2) $R_{x}^{t_{1}}\left(\tilde{L}^{\eta}\right) \subset^{s} \tilde{L}^{\eta}$ for $t_{1}=\rho\left(\tilde{L}^{\eta}, \tilde{L}^{\eta}\right)$.

Before we prove this proposition we need a lemma.

LEMMA 4.6. - Let $L \subset J$ be an interval, not containing a periodic orbit:

(1) If there exists $M>0$ such that $R_{x}^{s}(L) \cap L=\emptyset$ for all $s<M$ then there is $\varepsilon^{\prime}>0$ such that $s_{0}=\rho\left(L^{\varepsilon^{\prime}}, L^{\varepsilon^{\prime}}\right)>M$.

(2) Given any $c \in(0,1)$. If $M$ as above is such that $2 \lambda^{M}<c\left(1-\lambda^{\eta_{*}}\right)$, then there is $c^{\prime} \in(0, c)$ such that $t_{0}=\rho\left(L^{\varepsilon^{\prime}}, L^{c^{\prime} \varepsilon^{\prime}}\right)$ and $R_{x}^{t_{0}}\left(L_{\varepsilon^{\prime}}\right) \subset^{s} L_{c^{\prime} \varepsilon^{\prime}}$.

Proof. - Item (1). Fix some $M>0$ such that $R_{x}^{s}(L) \cap L=\emptyset$ for all $s \leqslant M$. Take $\varepsilon \in$ $\left(0, \varepsilon_{0}\right)$, set $s_{0}=\rho\left(L^{\varepsilon}, L^{\varepsilon}\right)>0$ and assume $s_{0}<M$. By hypothesis, $R_{x}^{s_{0}}(L) \cap L=\emptyset$; then there is $\varepsilon_{1}>0$ such that $R_{x}^{s_{0}}\left(L^{\varepsilon_{1}}\right) \cap L^{\varepsilon_{1}}=\emptyset$. Notice that $s_{1}=\rho\left(L^{\varepsilon_{1}}, L^{\varepsilon_{1}}\right)>s_{0}+\eta_{*}>s_{0}$. Repeating this argument a finite number of times we obtain some $\varepsilon^{\prime}:=\varepsilon_{k}>0$ and $\rho\left(L^{\varepsilon^{\prime}}, L^{\varepsilon^{\prime}}\right)>M$ which is what we wanted to prove.

Item (2). Take any $c \in(0,1)$. Since $2 \lambda^{M}<c\left(1-\lambda^{\eta_{*}}\right)$ there is $c_{1}>0$ such that $c_{1}+\frac{2 \lambda^{M}}{1-\lambda^{\eta *}}<c$. As in the previous item, let $s_{0}=\rho\left(L^{\varepsilon^{\prime}}, L^{\varepsilon^{\prime}}\right)>M$. We shall construct two sequences of numbers inductively, say $\left\{c_{i}, s_{i}\right\}_{i \in \mathbb{N}}$. $c_{1}$ is already given. Define for $i \geqslant 1: s_{i}=\rho\left(L^{\varepsilon^{\prime}}, L^{c_{i} \varepsilon^{\prime}}\right)$ and for $i \geqslant 2: c_{i}=\inf \left\{b>0 \mid R_{x}^{s_{i}-1}\left(L^{\varepsilon}\right) \subset^{s} L^{b \varepsilon}\right\}$.

Let $I=\min \left\{i \in \mathbb{N} \mid s_{i}=s_{i+1}\right\}$ (or equivalently $c_{i+1} \leqslant c_{i}$ ), with the convention $I=\infty$ if the set is empty. Observe that for $i<I$ we have $c_{i+1}>c_{i}$ and also $s_{i+1}<s_{i}$; since at the time we consider a wider box around $L$ it is possible we catch some previous returns to it. Notice $s_{1} \geqslant s_{0}$ since $c_{1}<c<1$. On the other hand, for $i<I$, we have $c_{i} \leqslant 2 \lambda^{s_{i-1}}+c_{i-1}$, that is the maximal stable width of the return plus the width of the $\varepsilon^{\prime} c_{i-1}$-box around $L$. Hence

$$
c_{i} \leqslant 2 \varepsilon \sum_{j=1}^{i-1} \lambda^{s_{j}}+c_{1} .
$$

Inductively, for all $i \in\{j, \ldots, I\}$ we have that $s_{j} \geqslant s_{0}$ and $c_{j}<c$. We claim that $c_{i}<c$ and hence, $s_{i} \geqslant s_{0}$ since $L^{c_{i} \varepsilon^{\prime}} \subset L^{\varepsilon^{\prime}}$. Since we have $s_{j}-s_{j+1}>0$, then $s_{j}-s_{j+1}>\eta_{*}$, and therefore, $s_{i-r} \geqslant s_{0}+(r-1) \eta_{*}$ for $r \in\{1, \ldots, i-1\}$. Hence,

$$
c_{i} \leqslant 2 \varepsilon \sum_{r=0}^{i-2} \lambda^{s_{0}+r \eta_{*}}+c_{1}<\frac{2 \varepsilon \lambda^{M}}{1-\lambda^{\eta_{*}}}+c_{1}<c,
$$

that is what we claimed.

Since $s_{0} \leqslant s_{1}$ and $s_{i}<s_{1}-i \eta_{*}$, there must be some $i$ such that $s_{i}=s_{i+1}$. Therefore $I<\infty$. Take $c^{\prime}=c_{I}$, and $t_{0}:=s_{I}=\rho\left(L^{\varepsilon^{\prime}}, L^{c^{\prime} \varepsilon^{\prime}}\right)$. 
COROLlary 4.7. - Denote by $\hat{L}=\left\{z \in L \mid L^{\varepsilon^{\prime}} \cap R_{x}^{t_{0}}\left(L^{\varepsilon^{\prime}}\right) \neq \emptyset\right\}$, and set $\tilde{L}=L \backslash \hat{L}$. Then $\tilde{L}$ is an interval and there is $c^{\prime \prime}>0$ such that $c^{\prime \prime} \in\left(c^{\prime}, c\right)$ and $R_{x}^{t_{1}}\left(\tilde{L}^{\varepsilon^{\prime}}\right) \subset^{s} \tilde{L}^{c^{\prime \prime} \varepsilon_{0}}$.

Proof. $-\tilde{L}$ is an interval since there are no periodic orbits passing through $J^{\varepsilon}$. To obtain the second part of the corollary denote by $d_{1}=c^{\prime}$ (from the previous lemma) and observe $r_{1}=\rho\left(\tilde{L}^{\varepsilon^{\prime}}, \tilde{L}_{d_{1} \varepsilon^{\prime}}\right)>t_{0}>M$. Then, we can apply the same arguments with the interval $\tilde{L}$ instead of $L$.

LEMMA 4.8. - If there is no periodic orbit on $J$ then for any $M>0$ there is a subinterval $L \subset J$ such that $R_{x}^{s}(L) \cap L=\emptyset$ for $s \leqslant M$.

Proof. - Let us suppose that for $0<s_{0}<M$, the first return of $J^{\varepsilon}$ into itself, happens that $R_{x}^{s_{0}}(J) \cap J \neq \emptyset$. Since there is no periodic orbit in $J$ we know one of the borders of $J$, say $J^{+}$, must return inside the box. We call $z \in R_{x}^{s_{0}}(J) \cap J$ the farthest point to $J^{+}$. Let $w \in J$ such that $R_{x}^{s_{0}}(w)=z \in J$.

It is important we verify $R_{x}^{s_{0}}\left(\left[w, J^{+}\right]^{\varepsilon}\right) \subset J^{\varepsilon}$. This will guarantee we do not lose any point of the box and so, no point of $\omega(I)$. Let $L=J \backslash\left[w, J^{+}\right]$and we seek for the first return of the box on $L$, that is $s_{1}>0$ such that:

$$
R_{x}^{s_{1}}\left(L^{\varepsilon}\right) \cap L^{\varepsilon} \neq \emptyset
$$

Observe now that $R_{x}^{s}(L) \cap L=\emptyset$ for $s \leqslant s_{0}+\eta_{*}$.

If now we can say $R_{x}^{s}(L) \cap L=\emptyset$ for $s<M$, we are done. However, if it does not happen we repeat the above construction on $L$, obtaining another subinterval $L^{\prime} \subset L \subset J$ for which

$$
R_{x}^{s}\left(L^{\prime}\right) \cap L^{\prime}=\varnothing ; \quad \forall s \leqslant s_{2}+\eta_{*}
$$

Since $s_{2} \geqslant s_{1}+\eta_{*} \geqslant s_{0}+2 \eta_{*}$ we are sure to reach $M$ in finite steps. Observe the way we cut the interval $J$ to obtain $L$ guarantee that any orbit of $J$ is traced by one of $L$.

Now, we can give a proof for Proposition 4.5.

Proof of Proposition 4.5. - Fix some $c<1$ and take $M>0$ big enough such that $2 \lambda^{M}<\left(1-\lambda^{\eta_{*}}\right)$. Using Lemma 4.8 set $L \subset J$ such that $R_{x}^{s}(L) \cap L=\emptyset$ for all $s \leqslant M$ and $\omega(L)=\omega(J)$. Now, Lemma 4.6 says there exists $\varepsilon_{0}>0$ such that $\rho\left(L^{\varepsilon_{0}}, L^{\varepsilon_{0}}\right)>M$ and there exists $0<c^{\prime}<c$ such that $R_{x}^{t_{0}}\left(L^{\varepsilon_{0}}\right) \subset^{s} L^{c^{\prime} \varepsilon_{0}}$ where $t_{0}=\rho\left(L^{\varepsilon_{0}}, L^{c^{\prime} \varepsilon_{0}}\right)$.

Moreover, on Corollary 4.7 we defined the set

$$
\hat{L}=\left\{z \in L \mid L^{\varepsilon} \cap R_{x}^{t_{0}}\left(L^{\varepsilon}\right) \neq \emptyset\right\} .
$$

Denote by $\tilde{L}=L \backslash \hat{L}$; and so, there is $c^{\prime \prime}>0$ such that $c^{\prime}<c^{\prime \prime}<c$ and $R_{x}^{t_{1}}\left(\tilde{L}^{\varepsilon_{0}}\right) \subset^{s} \tilde{L}^{c^{\prime \prime} \varepsilon_{0}}$, where $t_{1}=\rho\left(\tilde{L}^{\varepsilon_{0}}, \tilde{L}^{c^{\prime \prime} \varepsilon_{0}}\right)$.

Take $\eta=c^{\prime \prime} \varepsilon_{0}$. Since $c^{\prime}<c^{\prime \prime}<c$ we know that $L^{c^{\prime} \varepsilon_{0}} \subset L^{c^{\prime \prime} \varepsilon_{0}} \subset L^{\varepsilon_{0}}$ and then $\rho\left(L^{\varepsilon_{0}}, L^{c^{\prime} \varepsilon_{0}}\right)=\rho\left(L^{\varepsilon_{0}}, L^{c^{\prime \prime} \varepsilon_{0}}\right)=\rho\left(L^{\eta}, L^{\eta}\right)$. Hence we have found that

(1) $R_{x}^{t_{0}}\left(L^{\eta}\right) \subset^{s} L^{\eta}$ and $R_{x}^{t_{0}}\left(L^{\eta}\right) \cap \tilde{L}^{\eta}=\emptyset$, for $t_{0}=\rho\left(L^{\eta}, L^{\eta}\right)$.

(2) $R_{x}^{t_{1}}\left(\tilde{L}^{\eta}\right) \subset^{s} \tilde{L}^{\eta}$, where $t_{1}=\rho\left(\tilde{L}^{\eta}, \tilde{L}^{\eta}\right)$.

This is what we wanted to prove to conclude Proposition 4.5. 
Now, we are ready to construct an irrational torus and then finish the proof in case I. Let $D_{0}:=L^{\eta}$ and $t_{0}=\rho\left(D_{0}, D_{0}\right)$, given on Proposition 4.5. Recall $D_{0} \subset N_{x} \subset M$ for some $x \in \Lambda^{+}$. Moreover, we can think there is $\Sigma$, an extension of $N_{x}$ where $R_{x}^{t_{0}}\left(D_{0}\right) \subset \Sigma$. Define $P_{0}: D_{0} \rightarrow \Sigma$ as $P_{0}(\cdot)=R_{x}^{t_{0}}(\cdot)$. Recall that the orbit of no point of $D_{0}$ falls into a singularity. Since $R_{x}^{t_{0}}\left(D_{0}\right) \cap D_{0} \neq \emptyset$, there is a point $z \in D_{0}$ such that $P_{0}(z) \in D_{0}$. Therefore, we can extend the function $\tau_{x}^{t_{0}}(\cdot)$ to $D_{1}:=D_{0} \cup P_{0}\left(D_{0}\right)$. In this way we obtain an extension of $P_{0}$ called $P_{1}: D_{1} \rightarrow \Sigma$.

By induction, we can repeat this procedure until we find some $\tilde{n} \in \mathbb{N}$ such that

$$
D_{i} \cap \bigcup_{j \leqslant \tilde{n}} S_{j} \neq \emptyset
$$

where $S_{1}=\left\{X^{s}(y) \mid y \in D_{0}\right.$ and $\left.s \in\left[0, \tau_{x}^{s}(y)\right)\right\}$, and $S_{j}$ is defined inductively. If $D_{i} \cap S_{j} \neq \varnothing$ for $1<j<\tilde{n}$ then $D_{\tilde{n}-j} \cap S_{1} \neq \varnothing$. Take $n=\tilde{n}-j$. We can think $D_{n}$ is glued smoothly to $D_{0}$. In fact, this is possible since there is a function $\tau^{*}: D_{n} \cap S_{1} \rightarrow$ $\mathbb{R}^{-} \cup\{0\}$, the time a point on the intersection spent to flow back to $D_{0}$, that is: for $y \in D_{n} \cap S_{1}, X^{\tau^{*}(y)}(y) \in D_{0}$. Moreover, we can extend the domain of $\tau^{*}$ to some of the last $D_{n} \cup D_{n-1} \cup \cdots$, and glueing this function with the zero function defined on the first boxes of the sequence $D_{0} \cup D_{1} \cup \cdots$. Observe that $\bigcup_{i \leqslant n} \tau^{*}\left(D_{i}\right)$ is a connected smooth surface embedded in $M$. Observe the intersection of $D_{n}$ and $D_{0}$ corresponds to the first return of $\tilde{L}_{\eta}$ into itself, called $t_{1}$ in Proposition 4.5. Hence $D_{n} \subset^{s} D_{0}$, and more important, there is such $\tilde{n}$ in (5) and this process of extension stops.

By now, we restrict our attention to $D_{0}: D_{0}$ splits into three rectangles, $A \cup C \cup B$ defined as $A=\left(R_{x}^{t_{0}}\right)^{-1}\left(D_{0} \cap D_{1}\right), B=\left(R_{x}^{t_{1}}\right)^{-1}\left(D_{0} \cap D_{n}\right)$ and $C$ the region in $D_{0}$ in between $A$ and $B$. Denote by $G: D_{0} \rightarrow D_{0}$ the first return map of the flow to $D_{0}$ and notice that $G(A)=D_{0} \cap D_{1}$ and $G(B)=D_{0} \cap D_{n}$. Moreover, we notice also that $R_{x}^{t_{0}}(C) \subset D_{1}$ and $R_{x}^{t_{1}}(C) \subset D_{n}$. Denote by $\tilde{D}_{0}:=D_{0} \cup R_{x}^{t_{0}}(C) \cup R_{x}^{t_{1}}(C)$, and consider $\tilde{G}: \tilde{D}_{0} \rightarrow \tilde{D}_{0}$, the corresponding first return map. Observe that still $\tilde{G}(A)=D_{0} \cap D_{1}$ and $\tilde{G}(B)=D_{0} \cap D_{n}$. Also $\tilde{G}(C)=R_{x}^{t_{0}}(C)$ and $\tilde{G}\left(R_{x}^{t_{0}}(C)\right)=R_{x}^{t_{1}}(C)$; the only missing part is the image of $R_{x}^{t_{1}}(C)$. It is not difficult to see that the orbit of points in $C$ pass through $D_{0}$ near the inner border points of $\tilde{G}(A)$ and $\tilde{G}(B)$ (see Fig. 1)

LEMMA 4.9. - If there there is no periodic orbit passing through $D_{0}$ then $\left.\tilde{G}\right|_{R_{x}^{t_{1}}(C)}$ is continuous.

Proof. - If it is not the case, the rectangle $C$ splits into at least two sub-rectangles, call $C_{A}$ and $C_{B}$ those sharing part of its boundary with $A$ and $B$, respectively. Observe

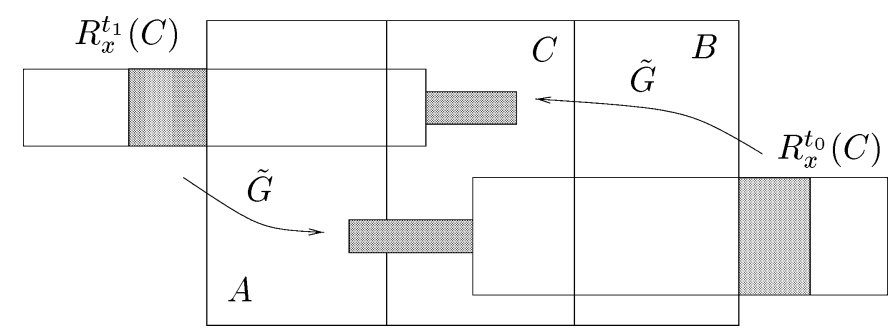

Fig. 1. 
$\tilde{G}\left(R_{x}^{t_{1}}\left(C_{A}\right)\right)=G\left(C_{A}\right)$ and is connected to $\tilde{G}(B)$, and $\tilde{G}\left(R_{x}^{t_{1}}\left(C_{B}\right)\right)=G\left(C_{B}\right)$ and is connected to $\tilde{G}(A)$. Note that in both cases the other stable boundary must reach the stable boundary of $D_{0}$. In fact, if not, we are missing some points of $D_{0}$ that actually passes in the formed gap, and this is not possible since $\tilde{G}$ is a true first return map to $\tilde{D}_{0}$.

It is not difficult to see that in this setting either $\left.\pi \circ G\right|_{A \cup B}: A \cup B \rightarrow J$ or $\left.\pi \circ G\right|_{C_{A} \cup C_{B}}: C_{A} \cup C_{B} \rightarrow J$ is onto. Therefore there exists $z \in J$ that $G(z) \in W_{\eta}^{s}(z)$, and hence we obtain a periodic orbit in $D_{0}$ which is a contradiction. Hence $\left.\tilde{G}\right|_{R_{x}^{t_{1}}(C)}$ is continuous and so, $\left.G\right|_{C}$ is too.

Therefore, the domain of $P_{n}$, the set $D_{0} \cup \cdots \cup D_{n}$, is an embedded ring. It is not difficult to verify that $P_{n}^{m}\left(D_{0} \cup \cdots \cup D_{n}\right) \rightarrow \tilde{S}$ as $m \rightarrow \infty$ where $\tilde{S}$ is an invariant circle. Finally note that the saturated of $\tilde{S}$ by the flow, $\mathcal{T}=\left\{X^{t}(y) \mid y \in \tilde{S}\right.$ and $\left.t \in \mathbb{R}\right\}$ is an embedded torus on $M$; hence $w(I) \subset w(J)=\mathcal{T}$. Moreover, $\left.P_{n}\right|_{\tilde{S}}$ corresponds to the Poincare first return map of $X^{t}$ to $\tilde{S}$. Since $P_{n}$ is a diffeomorphism of the circle without periodic points, it is conjugated to an irrational rotation (see [5]). Therefore, the restriction of $X^{t}$ to $\mathcal{T}$ is conjugated to a linear irrational flow on the torus. Here we conclude the proof of Proposition 4.2 in the case I.

Now we deal with the case II, that is: $J_{s}^{\varepsilon} \cap\left[R_{x}^{t-s}\left(J_{s}\right)\right]^{\varepsilon}=\emptyset$, for all $t, s \in \mathcal{P}$; but first, we shall exploit this disjointness property to measure the area of the strip defined by the family $\left\{J_{S}\right\}$.

Remark 2. - For any set $A \subset N_{x} \subset M$, define

$$
S(A)=\left\{X^{s}(z) \mid z \in A, s \in\left[-\frac{\eta_{*}}{2}, \frac{\eta_{*}}{2}\right]\right\} .
$$

There is a constant $K>0$ only depending on $\|X\|_{C^{1}}$ such that if $A=J^{\varepsilon}$, for some interval $J$ and $\varepsilon>0$ then $S(J):=S\left(J^{\varepsilon}\right)$ is such that $K \operatorname{Vol}(S(J)) \geqslant|J|$.

LEMMA 4.10. - For any $s \in \mathcal{P}$ we have that:

$$
\int_{0}^{\infty}\left|R_{x_{s}}^{t}\left(J_{s}\right)\right| \mathrm{d} t<+\infty
$$

and hence, $\left|R_{x_{s}}^{t}\left(J_{s}\right)\right| \rightarrow 0$ as $t \rightarrow \infty$.

Proof. - Fix some $s \in \mathcal{P}$ and denote by $J=J_{s}$ and $x=x_{s}$. Notice that for all $l, t \in \mathcal{P}$ such that $|l-t| \geqslant \eta_{*}$ we have:

$$
S\left(R_{x}^{l}(J)\right) \cap S\left(R_{x}^{t}(J)\right)=\emptyset .
$$

Let us calculate the integral $\int_{0}^{\infty}\left|R_{x}^{t}(J)\right| \mathrm{d} t$. For that, first observe $\int_{\mathcal{P}}\left|R_{x}^{t}(J)\right| \mathrm{d} t \leqslant$ $K \operatorname{Vol}(M)$. In fact, let $\mathcal{Q}$ be a partition of $\mathcal{P}$ of elements of diameter less than $\eta_{*}$. Remark 2, together with the disjointness property on (6) imply:

$$
\int_{\mathcal{P}}\left|R_{x}^{t}(J)\right| \mathrm{d} t \leqslant \sum_{Q \in \mathcal{Q}} \int_{Q}\left|R_{x}^{t}(J)\right| \mathrm{d} t \leqslant K \sum_{Q \in \mathcal{Q}} \operatorname{Vol}\left(S\left(R_{x}^{t_{Q}}(J)\right)\right) \leqslant K \operatorname{Vol}(M)
$$

where $t_{Q} \in Q \in \mathcal{Q}$. 
Now arrange the elements of $\mathcal{Q}$ in an ascending way, say $\mathcal{Q}=\left\{\left[a_{i}, b_{i}\right]\right\}, i \in \mathbb{N}$, such that $b_{i-1}<a_{1}$ and $\left(b_{i-1}, a_{i}\right) \cap Q=\emptyset$ for any $Q \in \mathcal{Q}$ and $i \in \mathbb{N}$. Notice that there exists $\tilde{\gamma}>0$ such that $\left|L^{a_{i}-s}\right|_{E\left(x_{s}\right)} \mid \geqslant \tilde{\gamma}^{a_{i}}$ for any $s \in\left(b_{i-1}, a_{i}\right)$; otherwise, there must be a point of $\mathcal{P}$ between $b_{i-1}$ and $a_{i}$, which is impossible. Using the fact that

$$
\left.\left.\left|L^{a_{i}-s}\right|_{E(x)}|| L^{a_{i}-s}\right|_{F(x)}\right|^{-1} \leqslant \lambda^{a_{i}-s}
$$

we obtain that $\forall t \in\left(0, a_{i}-b_{i-1}\right)$ :

$$
\left|L^{-t}\right| F\left(x_{a_{i}}\right) \mid \geqslant\left(\tilde{\gamma}^{-1} \lambda\right)^{t} .
$$

By a similar argument to the one we use in the proof of Lemma 4.1 there is $\mu \in(0,1)$ such that $\left|D_{z} R_{x_{a_{i}}}^{-t}\right| \tilde{F}(z) \mid<\mu^{t}$, for any $t \in\left(0, a_{i}-b_{i-1}\right), z \in R_{x}^{a_{i}}(J)$, where $\tilde{F}(z)=T_{z}\left(R_{x_{a_{i}}}^{a_{i}}(J)\right)$.

Hence,

$$
\left|R_{x_{a_{i}}}^{-t}\left(R_{x}^{a_{i}}(J)\right)\right|=\int_{R_{x}^{a_{i}}(J)}\left|D_{z}\left(R_{x_{a_{i}}}^{-t}\right)\right|_{\tilde{F}}\left|\mathrm{~d} z \leqslant \mu^{t}\right| R_{x}^{a_{i}}(J) \mid .
$$

Therefore,

$$
\int_{0}^{a_{i}-b_{i-1}}\left|R_{x}^{a_{i}-t}(J)\right| \mathrm{d} t \leqslant\left|R_{x}^{a_{i}}(J)\right| \int_{0}^{a_{i}-b_{i-1}} \mu^{t} \mathrm{~d} t \leqslant \frac{\left|R_{x}^{a_{i}}(J)\right|}{-\log \mu} .
$$

A similar argument of disjointness used to bound the integral over the hyperbolic times implies $\sum_{i}\left|R_{x}^{a_{i+1}}(J)\right|<K \operatorname{Vol}(M)$. Therefore,

$$
\int_{\mathbb{R}^{+}}\left|R_{x}^{t}(J)\right| \leqslant K \operatorname{Vol}(M)\left(1-\frac{1}{\log \mu}\right) .
$$

Finally, the function $\log \left|R_{x}^{t}(J)\right|$ is uniformly continuous on $t$; hence $\left|R_{x}^{t}(J)\right| \rightarrow 0$ as $t \rightarrow \infty$.

The following lemma measures how much the orbits of the flow $X^{t}$ differ from the orbits by $R^{t}$.

LEMMA 4.11. - There exists a constant $C>0$ such that if an interval $I \subset D_{x}^{t}$ for all $t \geqslant 0$ then, for any $y, w \in R_{x}^{r}(I)$, and $r, s \geqslant 0$ we have the following inequality:

$$
\left|\tau_{x_{r}}^{s}(y)-\tau_{x_{r}}^{s}(w)\right| \leqslant C \int_{r}^{\infty}\left|R_{x}^{t}(I)\right| \mathrm{d} t .
$$

Proof. - Recall that:

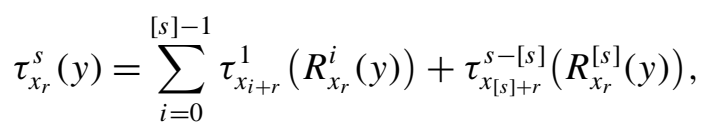


then

$$
\begin{aligned}
\left|\tau_{x_{r}}^{s}(y)-\tau_{x_{r}}^{s}(w)\right| \leqslant & \sum_{i=0}^{[s]-1}\left|\tau_{x_{i+r}}^{1}\left(R_{x_{r}}^{i}(y)\right)-\tau_{x_{i+r}}^{1}\left(R_{x_{r}}^{i}(w)\right)\right| \\
& +\left|\tau_{x_{[s]+r}}^{s-[s]}\left(R_{x_{r}}^{[s]}(y)\right)-\tau_{x_{[s]+r}-[s]}^{s-[}\left(R_{x_{r}}^{[s]}(w)\right)\right| .
\end{aligned}
$$

Now, the function $\tau^{1}$ is uniformly Lipschitz with some constant $C>0$. Hence,

$$
\leqslant C \sum_{i=0}^{[s]-1}\left|R_{x_{r}}^{i}\left(I_{r}\right)\right|+\left|R_{x_{r}}^{[s]}\left(I_{r}\right)\right| \leqslant C \int_{r}^{\infty}\left|R_{x_{0}}^{t}\left(I_{0}\right)\right| \mathrm{d} t
$$

and then the lemma follows.

Let us assume there are $t_{0}<t_{1}$, and $t_{0}, t_{1} \in \mathcal{P}$ such that $J_{t_{0}}^{\varepsilon} \cap J_{t_{1}}^{\varepsilon} \neq \emptyset$. In this case we shall obtain a sequence of times on which this intersection is at a small enough scale that allow us to control the limit. For that, call $Z=J_{t_{0}}^{\varepsilon} \cap J_{t_{1}}^{\varepsilon}$, and observe that $R_{x_{0}}^{t_{1}-t_{0}}(Z) \subset J_{t_{1}}^{\varepsilon}$, since $R_{x_{0}}^{t_{1}-t_{0}}\left(J_{t_{0}}^{\varepsilon}\right) \subset J_{t_{1}}^{\varepsilon}$. This implies the existence of certain $t^{*}>0$ such that $R_{x_{t_{1}}}^{t^{*}}\left(J_{t_{1}}^{\varepsilon}\right) \cap J_{t_{1}}^{\varepsilon} \neq \emptyset$. Remember it is necessary to fix a destination section any time we define some $R_{y}^{s}$. In this case we select the time $t^{*}$ in such a way that $x_{t^{*}}$ lie in some smooth extension of the section over $x_{t_{1}}$. Moreover, we can take this extension in such a way the tangent space on $x_{t^{*}}$ is exactly $\mathcal{N}_{x_{t}^{*}}$.

Let $t_{2}=t_{1}+t^{*}$ and set $C\left(t_{1}\right):=J_{t_{1}}^{\varepsilon}$. Notice that $C\left(t_{1}\right) \subset D_{x_{t_{1}}}^{t^{*}}$, then $R_{x_{t_{1}}}^{t_{2}-t_{1}}\left(C\left(t_{1}\right)\right) \cap$ $C\left(t_{1}\right) \neq \emptyset$, by construction. Now set $C\left(t_{2}\right):=R_{x_{t_{1}}}^{t_{2}-t_{1}}\left(C\left(t_{1}\right)\right)$. Inductively, take $t_{i+1}=$ $t_{i}+\tilde{t^{*}}$, such that $R_{x_{t_{i}}}^{t_{i+1}-t_{i}}\left(C\left(t_{i}\right)\right) \cap C\left(t_{i}\right) \neq \emptyset$, and denote $C\left(t_{i+1}\right):=R_{x_{t_{i}}}^{t_{i+1}-t_{i}}\left(C\left(t_{i}\right)\right.$ ) (see Fig. 2).

Notice that $\left|t_{i+1}-t_{i}\right|>\eta_{*}$, since any point must flow away certain amount of time before it returns into a neighborhood of it (Section 2.2). For any $j, i \in \mathbb{N}$ such that $j<i$, Lemma 4.11 implies that for each $s>0$ and $y \in R_{x_{t_{j}}}^{t_{i}-t_{j}}\left(J_{t_{j}}\right)$ we have that

$$
\left|\tau_{x_{t_{j}}}^{s}(y)-s\right| \leqslant C \int_{t_{i}-t_{j}}^{\infty}\left|R_{x_{t_{j}}}^{t}\left(J_{t_{j}}\right)\right| \mathrm{d} t
$$

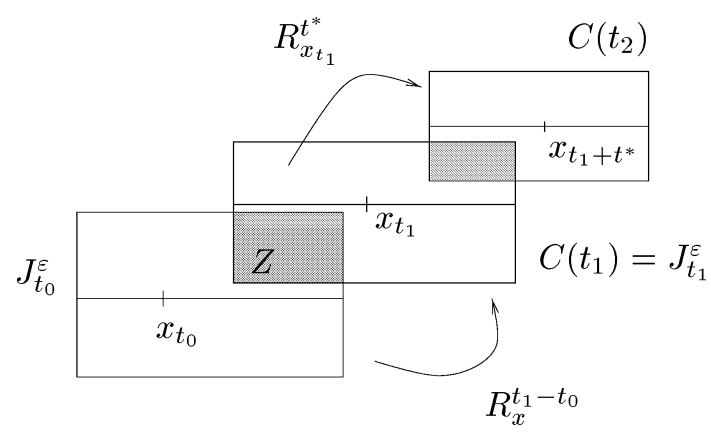

Fig. 2. 
Hence, for $j$ large enough we have that for all $y \in R_{x_{t_{j}}}^{t_{i}-t_{j}}\left(J_{t_{j}}\right)$,

$$
\left|\tau_{x_{j}}^{s}(y)-s\right| \leqslant \frac{\eta_{*}}{3}
$$

Fix $j \in \mathbb{N}$ such that also verifies that $C \varepsilon_{0} \lambda^{j} /(-\log (\lambda))<\eta_{*} / 3$. We may suppose $x_{t_{i}} \rightarrow z \in M$, as $i \rightarrow \infty$. Hence, Lemma 4.10 implies that $R_{x_{t_{j}}}^{t_{i}-t_{j}}\left(J_{t_{j}}\right)=J_{t_{i}} \rightarrow z$, as $i \rightarrow \infty$. Moreover, $R_{x_{t_{j}}}^{t_{i}-t_{j}}\left(C\left(t_{j}\right)\right) \rightarrow z$, as $i \rightarrow \infty$. However, the inequality on (7) only implies that

$$
\lim _{i \rightarrow \infty} X^{t_{i}-t_{j}}(y) \in\left\{X^{t}(z) \mid t \in\left[-\frac{\eta_{*}}{3}, \frac{\eta_{*}}{3}\right]\right\}
$$

for any $y \in J_{t_{j}}$. Moreover (8) is also valid for any $y \in R_{x_{t_{j}}}^{t_{j}-t_{1}}\left(J_{t_{1}}^{\varepsilon_{0}}\right)$ since

$$
\left|\tau_{x_{j}}^{s}(y)-s\right| \leqslant\left|\tau_{x_{j}}^{s}(y)-\tau_{x_{j}}^{s}(w)\right|+\left|\tau_{x_{j}}^{s}(w)-s\right|
$$

for any $y \in R_{x_{t_{1}}}^{t_{j}-t_{1}}\left(W_{\varepsilon_{0}}^{s}(\hat{w})\right)$, where $\hat{w} \in J_{t_{1}}$ and $R_{x_{t_{1}}}^{t_{j}-t_{1}}(\hat{w})=w$.

On the other hand, we know that $C\left(t_{j}\right) \cap C\left(t_{j+1}\right) \neq \emptyset$, that is

$$
R_{t_{1}}^{t_{j}-t_{1}}\left(J_{t_{1}}^{\varepsilon}\right) \cap R_{t_{1}}^{t_{j+1}-t_{1}}\left(J_{t_{1}}^{\varepsilon}\right) \neq \emptyset .
$$

This implies that there exists $\hat{w}_{1}, \hat{w}_{2} \in J_{t_{1}}$ such that

$$
R_{x_{t_{1}}}^{t^{*}}\left(W_{\varepsilon}^{s}\left(\hat{w}_{2}\right)\right) \subset W_{\varepsilon_{0}}^{s}\left(\hat{w}_{1}\right)
$$

since $W_{\varepsilon_{0}}^{c s}\left(\hat{w}_{k}\right)=W_{\varepsilon_{0}}^{s}\left(\hat{w}_{k}\right)$, for both, $k=1,2$; and hence, they are coherent because the center stable manifolds are dynamically defined.

Let $\hat{w}_{3}=R_{x_{t_{1}}}^{t^{*}}\left(\hat{w}_{2}\right) \in W_{\varepsilon_{0}}^{s}\left(\hat{w}_{1}\right)$. Denote by $w_{k}=R_{x_{t_{1}}}^{t_{j}-t_{1}}\left(\hat{w}_{k}\right) \in C\left(t_{j}\right)$, for $k=2,3$. Notice there is $s>\eta_{*}$ such that $X^{s}\left(w_{2}\right)=w_{3}$. Hence, $\lim _{i \rightarrow \infty} X^{t_{i}-t_{j}}\left(w_{3}\right)=X^{l}(z)$, where $|l|<\eta_{*} / 3$. On the other hand

$$
\lim _{i \rightarrow \infty} X^{t_{i}-t_{j}}\left(X^{s}\left(w_{2}\right)\right)=X^{l^{\prime}}\left(X^{s}(z)\right) \in\left\{X^{t}(z) \mid t \in\left[-\frac{\eta_{*}}{3}, \frac{\eta_{*}}{3}\right]\right\},
$$

since $s>\eta_{*} / 3$ it implies that $z$ is periodic.

Finally, if for any $t, s \in \mathcal{P}$ happens that $J_{s}^{\varepsilon} \cap J_{t}^{\varepsilon}=\emptyset$, by a similar calculation as in the proof the Lemma 4.10, we obtain

$$
\int_{\mathcal{P}}\left|J_{s}\right| \mathrm{d} s<+\infty \text { and hence, } \quad\left|J_{s}\right| \rightarrow 0 \quad \text { as } s \rightarrow \infty .
$$

Therefore, if we prove that there exists a sequence of times $\left\{s_{i}\right\}_{i \in \mathbb{N}}$ such that $\left|J_{s_{i}}\right|=\delta$, we get a contradiction. Now, like in Schwartz' proof of Denjoy's Lemma we have that for all $J_{s}$ maximal there exists $t>0$ such that $\left|R_{x_{s}}^{t}\left(J_{s}\right)\right|=\delta$. It is only left to verify that $t+s$ is a hyperbolic time. However, if it is not the case, like in the proof of Lemma 4.10 
the interval must increase its length contradicting the fact of the length being $\delta$. Here we finish the proof of Proposition 4.2.

Once we have proved Proposition 4.2, it is not difficult to verify the following corollary, regarding that only a finite number of irrational tori can exist on an admissible neighborhood of $\Lambda$ (see Section 3$)$.

COROLLARY 4.12. - If we assume that $\Lambda$ has a dominated splitting and it does not contain an irrational torus, there is $\delta_{0}>0$ and a neighborhood $V$ of $\Lambda$ where for any $(\delta, E)$-interval, $I \subset V$ with $\delta<\delta_{0}$, we have that $\omega(I) \subset \operatorname{Per}\left(\left.X^{t}\right|_{V}\right)$.

All these properties are still valid for $(\delta, F)$-intervals, considering the flow-time backwards.

\subsection{Dynamically defined local invariant manifolds}

Assume $\Lambda$ is a compact invariant set of $X^{t}$ with a dominated splitting, not containing an irrational torus. As we see in Corollary 4.12, there is $\delta_{0}>0$ such that any $(\delta, E)$ interval, $\delta<\delta_{0}$ has $\omega(I) \subset \operatorname{Per}\left(\left.X^{t}\right|_{V}\right)$ for some appropriate admissible neighborhood $V$.

LEMMA 4.13. - There exists $\delta_{3} \in\left(0, \delta_{0}\right)$ such that if any periodic point $p \in \Lambda$ has one of its connected components of $W^{u}(p)-\{p\}$, say $W$, such that $\left|R_{p}^{t}(W)\right| \leqslant \delta_{3}$ for all $t \geqslant 0$, then the other endpoint which is not $p$ is periodic and it is not hyperbolic of saddle type. Moreover, it is a sink or a non-hyperbolic periodic point.

Proof. - Let $U_{1} \supset \Lambda$ be a neighborhood where it is defined an extension of the invariant cone field of $\Lambda$. Let $\delta_{3}<\delta_{0}$ be a positive number such that any ball of radius $\delta_{3}$ centered on a point of $\Lambda$ is contained in $U$. Take a periodic point $p$ and $W$ as in the hypothesis of the lemma. There is a number $t_{p}>0$ such that $R_{p}^{t_{p}}(W)=W$. In fact, $t_{p}$ is the period of $p$ or twice. Notice that $W$ is a $\left(\delta_{3}, E\right)$-interval. Since $q \in V_{1}$ it is well defined the direction $F(q)=T_{q}(W)$ and $\left|L^{t_{q}}\right|_{F(q)} \mid \leqslant 1$ and hence, by the domination condition we have that $\left|L^{t_{q}}\right| E(q) \mid \leqslant \lambda<1$. Hence, if $p$ is hyperbolic it must be a sink.

The following lemma is a fundamental application of Proposition 4.2.

LEMMA 4.14. - For all $\varepsilon \in\left(0, \delta_{0}\right)$ there is $\gamma=\gamma(\varepsilon)>0$ such that:

(1) For all $t>0$ we have that:

(a) $R_{x}^{-t}\left(W_{\gamma}^{c u}(x)\right) \subset W_{\varepsilon}^{c u}\left(x_{-t}\right)$.

(b) $R_{x}^{t}\left(W_{\gamma}^{c s}(x)\right) \subset W_{\varepsilon}^{c s}\left(x_{t}\right)$.

(2) Given $\tilde{\gamma} \in\left(0, \gamma\left(\delta_{0}\right)\right)$ we have:

(a) $\left|R_{x}^{-t}\left(W_{\tilde{\gamma}}^{c u}(x)\right)\right| \rightarrow 0$ as $t \rightarrow \infty$, or $x \in W^{u}(p)$ for some periodic point $p$ and $p \in W_{\tilde{\gamma}}^{c u}(x)$.

(b) $\left|R_{x}^{t}\left(W_{\tilde{\gamma}}^{c s}(x)\right)\right| \rightarrow 0$ as $t \rightarrow \infty$, or $x \in W^{s}(p)$ for some periodic point $p$ and $p \in W_{\tilde{\gamma}}^{c s}(x)$.

Proof. - We shall only prove the statement for central unstable manifold since the same argument works for the central stable manifolds. For item 1 take $\delta_{0}$ from 
Proposition 4.2 and let $\varepsilon<\delta_{0}$. Recall that Corollary 3.3 assert there is $0<\delta<\delta_{0}$ such that if: $\left|R^{-s}\left(W_{\varepsilon}^{c u}(x)\right)\right|<\delta$ for $s \in[0, t]$, then $R^{-s}\left(W_{\varepsilon}^{c u}(x)\right) \subset W_{\varepsilon}^{c u}\left(x_{-s}\right)$ for $s \in[0, t]$. Arguing by contradiction, assume the statement of item (1) does not hold. Then there exist a sequence of numbers $\gamma_{n} \rightarrow 0$, points $x^{n} \in \Lambda$ and times $m_{n} \rightarrow \infty$ such that $\left|R^{-m_{n}}\left(W_{\gamma_{n}}^{c u}\left(x^{n}\right)\right)\right|>\delta$. We should be more precise on how this bound is exceeded; that is, $\left|R^{-s}\left(W_{\gamma_{n}}^{c u}\left(x^{n}\right)\right)\right| \leqslant \delta$ for $s \in\left(0, m_{n}\right)$, and $\left|R^{-m_{n}}\left(W_{\gamma_{n}}^{c u}\left(x^{n}\right)\right)\right|=\delta$. Let us write $I_{n}=R^{-m_{n}}\left(W_{\gamma_{n}}^{c u}\left(x^{n}\right)\right)$. At least for a subsequence, we can assume that $y^{n}:=X^{-m_{n}}\left(x^{n}\right) \rightarrow z \in \Lambda$ and also $I_{n}$ converges to some interval $I$ transversal to the $E$ direction. Notice $|I| \leqslant \delta$ and $z \in \operatorname{cl}(I)$. By construction: $I \subset \operatorname{Dom}\left(R^{t}\right)=V_{t}$ for all $t \geqslant 0$ and $\left|R^{t}(I)\right| \leqslant \delta_{0}$ for all $t \geqslant 0$. Hence is a $(\delta, E)$-interval. Now, since $z \in \operatorname{cl}(I)$ we know $\omega(z)=p \in \operatorname{Per}\left(X^{t}\right)$ as Proposition 4.2 says. On the other hand, $p$ is hyperbolic since $p \in \Lambda$. Therefore $z \in W^{s}(p)$. Observe that one connected component of $W^{u}(p) \backslash\{p\}$ has length less than $\delta$. In fact, $W^{u}(p)$ is the limit of the intervals $\left|I_{n}\right| \leqslant \delta$ and $z \in \operatorname{cl}(I)$. Hence, the previous lemma implies the other end point must be a periodic point and not an hyperbolic saddle. It may happen that $I_{n} \cap W^{s}(p) \neq \emptyset$ for $n>>1$. In this case we get a contradiction with the Inclination Lemma, since this intersection is transversal and $R^{m_{n}}\left(I_{n}\right)=R^{-m_{n}}\left(R^{m_{n}}\left(W_{\gamma_{n}}^{c u}\left(x^{n}\right)\right)\right) \rightarrow 0$ as $n \rightarrow \infty$. Hence, $I_{n} \cap W^{s}(p)=\emptyset$ for $n$ arbitrarily large. In this case, $\omega\left(y^{n}\right) \subset \Lambda$ is the other endpoint of the connected component of $W^{u}(p) \backslash\{p\}$ of length less than $\delta$. Then $\omega\left(y^{n}\right)$ is a periodic sink or a non-hyperbolic periodic point. This is a contradiction since all periodic points in $\Lambda$ are hyperbolic of saddle type, concluding the proof of item (1).

To prove item (2): Take $\delta<\delta\left(\gamma_{0}\right)$, and let $x \in \Lambda$ be a point such that there exists $\eta>0$ and a sequence of times $t_{k} \rightarrow \infty$ in such a way $\left|R^{-t_{k}}\left(W_{\gamma}^{c u}(x)\right)\right| \geqslant \eta$. Last item guarantees that $\eta<\delta_{0}$, since $\left|R^{-t}\left(W_{\gamma}^{c u}(x)\right)\right| \leqslant \delta_{0}$ for any $t \geqslant 0$. As in the previous case, we can obtain a sequence of intervals $I_{k}:=R^{-t_{k}}\left(W_{\gamma}^{c u}(x)\right)$ converging to some $(\eta, E)$-interval called $I$, where $z \in \operatorname{cl}(I)$, and $z \in \Lambda$. Hence $z \in W^{s}(p)$ for some periodic point $p \in \Lambda$ of saddle type. Observe $I$ is transversal to $W^{s}(p)$, even in the case $z \notin \operatorname{Int}(I)$. Avoiding a contradiction with the Inclination Lemma we have that $\left|W^{u}(p)\right| \leqslant \eta \leqslant \delta_{0}$. If $z \in \operatorname{Int}(I)$, as $y_{k}:=X^{t_{k}}(x) \rightarrow z$ we conclude $y_{k} \in W^{s}(p)$. Otherwise we get a contradiction since both connected components of $W^{u}(p) \backslash\{p\}$ have length less than $\delta_{0}$, and hence $\omega(x)$ must be a sink or a non-hyperbolic periodic point. Suppose $z \neq p$. Then for $k>>1$ all $y_{k}=X^{-t_{k}}(x)$ are contained in some fundamental neighborhood of $W^{s}(p)$. This is a contradiction, since $W^{s}(p)$ do not have self-intersections. Hence $z=p$. Therefore $y_{k} \in W_{\mathrm{loc}}^{s}(p)$. Otherwise $\omega(x)$ must be a sink or a non-hyperbolic periodic point. Hence, $x=p$, proving item (2) in case $z \in \operatorname{int}(I)$.

It is left to verify the case when $z \notin \operatorname{int}(I)$. Observe: $R^{-t_{k}}\left(W_{\gamma}^{c u}(x)\right) \cap W^{s}(p)=I_{k} \cap$ $W^{s}(p)$ and this intersection is not empty; otherwise, we find a contradiction in a similar way as in the proof of item (1). Combining the following two facts: $\left|R^{t}\left(R^{-t_{k}}\left(W_{\gamma}^{c u}(x)\right)\right)\right| \leqslant \delta_{0}$ for $t \in\left(0, t_{k}\right)$ and $X^{-t_{k}}(x) \rightarrow z$ as $k \rightarrow \infty$ we conclude $x \in W^{u}(p)$. Moreover $x \in W_{\delta_{0}}^{u}(p)$. If $p \notin W_{\gamma}^{c u}(x)$, as $y_{k} \rightarrow p$ when $k \rightarrow \infty$ and $\left|R^{-t_{k}}\left(W_{\gamma}^{c u}(x)\right)\right|$ do not converges to 0 we have that $R^{-t_{k}}\left(W_{\gamma}^{c u}(x)\right) \cap W^{s}(p) \neq\{p\}$ for $k>>0$, and in particular $I \subset W^{s}(p)$, a contradiction. Hence $p \in W_{\gamma}^{c u}(x)$, and we are done. 


\section{Proof of Main Lemma}

In this section we shall give a proof for the Main Lemma, stated on Section 3. Before we do that, some effort will be spent constructing two-dimensional boxes around certain points with nice properties of recurrence. These boxes shall allow us to treat the problem in a very similar way as in the case of diffeomorphisms on surfaces. On the other hand, under the hypothesis of the Main Lemma, we shall obtain more dynamical properties on the central stable and central unstable manifolds. Let us begin defining the standard box around a point in $\Lambda$; when $\Lambda$ has a dominated splitting, and the notion of distortion along the $c s$-direction on such boxes.

Let $x \in \Lambda$ be given. For any two intervals $J \subset W_{\gamma}^{c u}(x)$ and $T \subset W_{\gamma}^{c s}(x)$, both containing the point $(x, 0) \in \mathcal{N}_{x}$, we call the box, or $(J, T)$-box, around $x$ the set $B_{T}(J)=\exp _{x}(T \times J)$, with boundaries: $\partial^{c s}\left(B_{T}(J)\right)=\exp _{x}(\partial T \times J)$ and $\partial^{c u}\left(B_{T}(J)\right)=$ $\exp _{x}(T \times \partial J)$. Observe that if the size of the box, say $|T|+|J|$ is small enough, then $\partial^{c s}\left(B_{T}(J)\right)$ is transversal to the $E$-direction and also $\partial^{c u}\left(B_{T}(J)\right)$ is transversal to the $F$-direction. Also we can assume that for any $y \in \Lambda, \partial^{\alpha}\left(B_{T}(J)\right) \cap W_{\gamma}^{\alpha}(y)$ are relative open sets for $\alpha=c s$, cu. If $B_{T}(J)$ is a box and $x \in T^{\prime} \subset T$, we say $B_{T^{\prime}}(J)$ is a sub-box of $B_{T}(J)$ if it is a box and $\partial^{c s} B_{T^{\prime}}(J) \subset \partial^{c s} B_{T}(J)$. For simplicity, we shall denote only $B_{\varepsilon}(J)$, when $T \subset W_{\varepsilon}^{c s}(x)$.

DEFINITION 4. $-A$ box $B_{\varepsilon}(J)$ has distortion $C>0$, or precisely distortion along the cs-direction, if for any two intervals $J_{1}, J_{2} \subset B_{\varepsilon}(J)$, both transversal to the $E$ direction and whose end points lie in $\partial^{c s} B_{\varepsilon}(J)$ happens that:

$$
\frac{1}{C} \leqslant \frac{l\left(J_{1}\right)}{l\left(J_{2}\right)} \leqslant C .
$$

An equivalent way to obtain such distortion is by means of a projection along some foliation close to the $E$-direction defined on the box. Precisely, let $\mathcal{F}^{c s}$ be the foliation obtained by integrating some $C^{1}$ vector field $Y$ defined on $B_{\varepsilon}(J)$ such that $Y(p) \in C_{a}^{E}(p)$, for all $p \in \operatorname{int}\left(B_{\varepsilon}(J)\right)$ and for $p \in \partial^{c u}\left(B_{\varepsilon}(J)\right), Y(p) \in T_{p} \partial^{c u}\left(B_{\varepsilon}(J)\right)$. If we denote by $\Pi=\Pi\left(J_{1}, J_{2}\right)$ the projection along the leaves of $\mathcal{F}^{c s}$ between the two intervals $J_{1}, J_{2}$, then the box $B_{\varepsilon}(J)$ has distortion $C$ if there exists $C>0$ such that $1 / C \leqslant\left\|\Pi^{\prime}\right\| \leqslant C$.

In [21] they guarantee distortion on a dynamical box if it is provided of some bound on the sum of the length of the pre-images of the leaves of the foliation on the box. This is still valid in our context, since the generalized Poincaré transformation, behaves like a diffeomorphism (see Corollary 3.5 of [21]), and it can be stated as follows:

COROLlaRY 5.1. - There exists $\tau\left(<\delta_{2}\right)$ such that if for some $z \in B_{\varepsilon}(J) \cap \Lambda$ and $n>0$ we have

$$
\left\|L_{E\left(z_{-t}\right)}^{t}(z)\right\|<\lambda_{1}^{t}, \quad \text { for } 0 \leqslant t \leqslant n
$$

and the box $B(n):=B\left(R_{z}^{-n}(J(z))\right)$ satisfies that:

(1) $R_{z-n}^{n}(B(n)) \subset B_{\varepsilon}(J)$ and $R_{z}^{n}\left(\partial^{c s} B(n)\right) \subset \partial^{c s} B_{\varepsilon}(J)$,

(2) $R_{z-n}^{t-n}\left(R_{z}^{-n}(J(z))\right)$ has diameter less than $\tau$, for $0 \leqslant t \leqslant n$, then there exists $C_{1}$ such that $B\left(R_{z}^{-n}(J(z))\right)$ has distortion $C_{1}$. 
Using this corollary, we can prove more dynamical properties for central manifolds.

LEMma 5.2. - Assume $\Lambda$ is transitive and every proper compact invariant subset of $\Lambda$ is hyperbolic, then either $\left|R_{x}^{-t}\left(W_{\gamma}^{c u}(x)\right)\right| \rightarrow 0$ as $t \rightarrow \infty$ for any $x \in \Lambda$ or the direction $F$ is expanding (that is, $\left|L^{-t}\right| F(x) \mid \rightarrow 0$ as $t \rightarrow \infty$ ).

Proof. - Observe $\Lambda$ is not a periodic orbit of the flow. Suppose there is $x \in \Lambda$ such that limsup $\left|R_{x}^{-t}\left(W_{\gamma}^{c u}(x)\right)\right|>0$. Lemma 4.14 implies there is a periodic orbit $p \in \Lambda$ such that one of the components of $W^{u}(p)-\{p\}$ has length less than $\delta_{0}$. Also Lemma 4.13 implies the endpoint of this component $q \neq p$, is a sink or a non-hyperbolic periodic point, and so, $q \notin \Lambda$.

Since $\Lambda$ is transitive and non-trivial, there is $x_{0} \in \Lambda \backslash \operatorname{Per}\left(X^{t}\right)$ such that $x_{0} \in W^{s}(p)$. Moreover, there is a small neighborhood of $x_{0}$, call it $U \subset \mathcal{N}_{p}$ divided into two components by $W^{c s}\left(x_{0}\right) \subset W^{s}(p)$, where on one side, say the "upper" one, we have the same $\omega$-limit as $q$.

Take $\lambda<\lambda_{1}<\lambda_{2}<\lambda_{3}<1$, and $c>0$ such that $\lambda \lambda_{1}^{-1}<\lambda_{2}$ and $(1+c) \lambda_{2}<\lambda_{3}$. Take $\tilde{\delta}>0$ such that if $I$ is a $(\delta, E)$-interval on $z$ for $\delta<\tilde{\delta}$, then for all $y \in I$ we have:

$$
(1-c)^{t} \leqslant \frac{\left|L^{t}\right|_{F(y)} \mid}{\left|D R_{y}^{t} \tilde{F}_{\tilde{F}(y)}\right|} \leqslant(1+c)^{t}
$$

where $\tilde{F}(y)=T_{y} I$.

Let $B_{\varepsilon}(J)$ be a box for $x_{0}$ contained in $U$ and even so small such that

$$
R_{x_{0}}^{t}\left(B_{\varepsilon}\left(J^{+}\right)\right) \cap B_{\varepsilon}\left(J^{+}\right)=\emptyset, \quad \forall t \neq 0
$$

and $\left|R_{x_{0}}^{-t}(J(y))\right| \leqslant \delta$, for any $y \in B_{\varepsilon}(J) \cap \Lambda$ and $t \geqslant 0$; where $\delta+\varepsilon<\eta<\tau$ ( $\tau$ from Corollary 5.1 and $\eta$ the size of the box).

For any given $z \in B_{\varepsilon}(J) \cap \Lambda$ denote by $J^{+}(z)=J(z) \cap B_{\varepsilon}\left(J^{+}\right)$and for it we have:

$$
R_{z}^{-t}\left(J^{+}(z)\right) \cap B_{\varepsilon}\left(J^{+}\right)=\emptyset, \quad \forall t>0 .
$$

Let $B_{\varepsilon_{1}}(J)$ be a sub-box for a positive $\varepsilon_{1}<\varepsilon / 4$. We claim there is $K>0$ such that for any $z \in B_{\varepsilon_{1}}(J) \cap \Lambda$ :

$$
\int_{t \geqslant 0}\left|R^{-t}\left(J^{+}(z)\right)\right| \mathrm{d} t \leqslant K
$$

for any $z \in B_{\varepsilon_{1}}(J) \cap \Lambda$.

Once established this claim, the argument on the proof of Lemma 3.5.2 of [21] concludes the thesis of the lemma.

Let us prove the claim. Fix an arbitrary point $z \in B_{\varepsilon_{1}}(J) \cap \Lambda$. And consider the set

$$
M_{z}=\left\{\left.m \in \mathbb{R}^{+}|| L^{t}\right|_{E\left(z_{-m}\right)} \mid \leqslant \lambda_{2}^{t}, \forall t \in[0, m]\right\} .
$$

Corollary 5.1 implies that for any $m \in M_{z}$ the box

$$
B(m)=B_{\varepsilon_{1}}\left(R_{z}^{-m}\left(J^{+}(z)\right)\right)
$$


has distortion $C$. In fact, it is true that $R_{z_{-m}}^{m_{i}}(B(m)) \subset B_{\varepsilon}(J)$ and $R_{z_{-m}}^{m_{i}}\left(\partial^{c u} B\left(m_{i}\right)\right) \subset$ $\partial^{c u} B_{\varepsilon}(J)$. Notice also that for $m, n \in M_{z}, m \neq n$, then $B(m) \cap B(n)=\emptyset$. Take $\varepsilon_{2}=\varepsilon_{1} / 4$. For each $y \in \Lambda$ consider the three dimensional cube (defined on Section 2):

$$
S(y):=S\left(B_{\varepsilon_{2}}\left(W_{\gamma / 2}^{c u}(y)\right)\right) .
$$

Since $\Lambda$ is compact, it can be covered by a finite number of these cubes:

$$
\Lambda \subset \bigcup_{k=0}^{k_{0}} S\left(y_{k}\right) .
$$

For each $m \in M_{z}$, the point $z_{-m}$ belongs to some $S\left(y_{k}\right)$. Denote by $J(m):=B(m) \cap$ $S\left(y_{k}\right)$. If the size of the box $\eta$ is small enough, $J(m)$ is a non-empty interval contained in $B(m)$. Notice there are constants $K_{1}, K_{2}>0$ only depending on $\eta_{*}$ such that

$$
|J(m)| \leqslant K_{1}\left|W_{\gamma / 2}^{c u}\left(y_{k}\right)\right| \leqslant K_{2} \operatorname{Vol}\left(S\left(y_{k}\right)\right) .
$$

Since we have distortion $C$ on $B(m)$, we have

$$
\frac{1}{C} \leqslant \frac{|J(m)|}{\left|R_{z}^{-m}\left(J^{+}(z)\right)\right|} \leqslant C
$$

and hence,

$$
\left|R_{z}^{-m}\left(J^{+}(z)\right)\right| \leqslant C|J(m)|
$$

Therefore,

$$
\int_{M_{z}}|J(t)| \mathrm{d} t \leqslant K \sum_{k=0}^{k_{0}} \int_{-\eta_{*} / 2}^{\eta_{*} / 2}\left|X^{t}\left(W_{\gamma / 2}^{c u}\left(y_{k}\right)\right)\right| \mathrm{d} t \leqslant K \operatorname{Vol}(M) .
$$

Now we have to bound the integral in the gaps of $M_{z}$. Let $M, m \in M_{z}$ such that $M>m$ and $M_{z} \cap(m, M)=\emptyset$ (perhaps $\left.M=\infty\right)$. For $t \in(m, M)$, we have that $\left|L^{t}\right|_{E\left(z_{-m-t}\right)} \mid \geqslant \lambda_{1}^{t}$. Then, the dominated splitting condition implies:

$$
\left|L^{-t}\right|_{F\left(z_{-m}\right)} \mid \leqslant\left(\lambda \lambda_{1}^{-1}\right)^{t} \leqslant \lambda_{2}^{t}
$$

and hence,

$$
\left|D R_{y}^{-t}\right|_{\tilde{F}(y)} \mid \leqslant \lambda_{3}^{t}
$$

where $\tilde{F}(y)=T_{y} R_{z}^{-m}\left(J^{+}(z)\right)$. Denote $J_{m}=R_{z}^{-m}\left(J^{+}(z)\right)$, then

$$
\int_{m}^{M}\left|R^{t}\left(J_{m}\right)\right| \mathrm{d} t \leqslant \frac{1}{1-\lambda_{3}}\left|J_{m}\right| .
$$

Hence, 


$$
\begin{aligned}
\int_{t \geqslant 0}\left|R^{t}\left(J^{+}(z)\right)\right| \mathrm{d} t & \leqslant \int_{M_{z}}\left|R^{t}\left(J^{+}(z)\right)\right| \mathrm{d} t+\sum_{j} \int_{m_{j}}^{M_{j}}\left|R^{t}\left(J_{m_{j}}\right)\right| \mathrm{d} t \\
& \leqslant K \operatorname{Vol}(M)+\frac{1}{1-\lambda_{3}} \sum_{j}\left|J_{m_{j}}\right| .
\end{aligned}
$$

where $\left\{\left(m_{j}, M_{j}\right)\right\}$ denotes all the gaps of $M_{z}$. However, $m_{j} \in M_{z}$ and hence, the box $B\left(m_{j}\right)$ has distortion $C$. Hence, we can bound the last sum above $\sum_{j}\left|J_{m_{j}}\right| \leqslant C \operatorname{Vol}(M)$, proving the claim.

An analogous result holds also for center stable manifolds, allowing us to conclude that for all $x \in \Lambda$ we have both, as $t \rightarrow \infty$ :

$$
\left|R_{x}^{-t}\left(W_{\gamma}^{c u}(x)\right)\right| \rightarrow 0 \quad \text { and } \quad\left|R_{x}^{t}\left(W_{\gamma}^{c s}(x)\right)\right| \rightarrow 0 .
$$

In the sequel, we shall take $\gamma \leqslant \gamma_{1}$.

COROLLARY 5.3. - Given $\varepsilon>0$ there exists some $t_{0} \geqslant 0$ such that for any $x \in \Lambda$ and $t \geqslant t_{0}$ we have:

$$
\left|R_{x}^{-t}\left(W_{\gamma}^{c u}(x)\right)\right| \leqslant \varepsilon \quad \text { and } \quad\left|R_{x}^{t}\left(W_{\gamma}^{c s}(x)\right)\right| \leqslant \varepsilon
$$

\subsection{Returns}

Let $B_{\varepsilon}(J)$ be a box and suppose $x \in J \cap \Lambda$. Recall that for any $y \in B_{\varepsilon}(J) \cap \Lambda$ we have defined $J(y)=W_{\gamma}^{c u}(y) \cap B_{\varepsilon}(J)$. Given a neighborhood $V$, we consider the set of points whose entire orbit stays on $V$ and they are asymptotic to $\Lambda$

$$
\tilde{\Lambda}=\left\{y \in V \mid \operatorname{dist}\left(\Phi^{t}(x), \Lambda\right) \rightarrow 0 \text { as } t \rightarrow \pm \infty\right\} .
$$

Notice that $\tilde{\Lambda}$ also has dominated splitting since it is contained in $V$. Although it is not compact, the arguments in Lemmas 4.14 and 5.2 apply, and so, there are central stable and central unstable manifolds on points of $\tilde{\Lambda}$ dynamically defined.

Definition 5. - For $\delta>0$, we say the box $B_{\varepsilon}(J)$ is $\delta$-adapted if for every $y \in$ $B_{\varepsilon}(J) \cap \Lambda$ the following conditions are satisfied:

(1) $\left|R_{x}^{-t}(J(y))\right| \leqslant \delta$ for all $t \geqslant 0$,

(2) $R_{x}^{-t}(J(y)) \cap B_{\varepsilon}(J)=\emptyset$ or $R_{x}^{-t}(J(y)) \subset B_{\varepsilon}(J)$, for all $t \geqslant 0$.

Before we prove the existence of adapted boxes for certain points on $\Lambda$, we need to recognize some configuration that implies the presence of periodic orbits in a box, as the following lemma states.

LEMmA 5.4. - Let $J \subset W_{\gamma}^{c u}(x)$ be an interval, for some $x \in \Lambda$ and assume $W_{\gamma}^{c u}(x)$ is dynamically defined. Suppose there is $t>0$ such that $R_{x}^{-t}(J) \cap J \neq \emptyset$. Then, the $\alpha$-limit of $x$ is a periodic orbit. Hence $x$ is periodic.

This lemma is also true replacing $c u$ by $c s, \alpha$-limit by $\omega$-limit and takeing positive iterations. 
Proof. - Suppose there is $t>0$ such that $R_{x}^{t}(J) \cap J \neq \emptyset$, then $x_{t} \in W_{\gamma}^{c u}(x)$. Therefore there is a sequence $t_{k} \rightarrow \infty$ such that $x_{t_{k}} \in W_{\gamma}^{c u}(x)$ and that $x_{t_{k}} \rightarrow x$ as $k \rightarrow \infty$. Since $W_{\gamma}^{c u}(x)$ is dynamically defined, for $k$ big enough: $R_{x}^{-t_{k}}\left(W_{\gamma}^{c u}(x)\right) \subset W_{\gamma}^{c u}(x)$, and hence $\alpha(x)$ is a periodic orbit. Moreover $x$ is periodic.

LEMMA 5.5. - For any $\delta$ small and any point $x \in \Lambda \backslash \operatorname{Per}\left(X^{t}\right)$ there exists $\delta$-adapted boxes associated to $x$.

Proof. - Let $x \in \Lambda$ be a non-periodic point. Notice that condition (1) of the definition is easily satisfied if the height of the box is small enough. So, we are only interested on condition (2). In spite of the previous lemma, there is an interval $J \subset W_{\gamma}^{c u}(x)$ for which $\Phi^{-t}(J) \cap J=\emptyset$, for any $t>0$.

Given some order relation on $J$ we can split $J$ into two intervals according to $x$, say $J^{+}=\{y \in J \mid y>x\}$ and $J^{-}=\{y \in J \mid y<x\}$. Now we define

$$
A^{+}=\left\{y \in J^{+} \mid \exists z \in \tilde{\Lambda}, W_{\gamma}^{c s}(z) \cap J^{+}=\{y\}\right\}
$$

and the corresponding set for $J^{-}$. Let $\left\{\varepsilon_{k}>0\right\}_{k \in \mathbb{N}}$ be a decreasing sequence of numbers converging to 0 , and let $B_{\varepsilon_{k}}$ be a sequence of boxes around $J$ such that $B_{\varepsilon_{k+1}} \subset B_{\varepsilon_{k}}$ and $\bigcap_{k} B_{\varepsilon_{k}}=J$. For any $y \in A^{+}$and any positive integer $k$ we denote by $U\left(y, \varepsilon_{k}\right)$ the connected component of $W_{2 \gamma}^{c u}(z) \cap B_{\varepsilon_{k}}(J)$ which contains $y$. Consider also

$$
\begin{gathered}
I\left(y, \varepsilon_{k}\right)=\left\{(z, n) \mid z \in B_{\varepsilon_{k}}(J) \cap \Lambda, n>0\right. \text { and such that } \\
\left.R_{x}^{-n}(J(z)) \cap U\left(y, \varepsilon_{k}\right) \neq \emptyset\right\} .
\end{gathered}
$$

If there exist points $y^{+} \in J^{+}$and $y^{-} \in J^{-}$, and there is an integer $k$ with \#I $\left(y^{ \pm}, \varepsilon_{k}\right)=$ 0 then the box $B_{\varepsilon_{k}}\left(J^{\prime}\right)$ around $J^{\prime}=\left(y^{-}, y^{+}\right)$is $\delta$-adapted. Notice $\partial^{c s} B_{\varepsilon_{k}}\left(J^{\prime}\right)=$ $U\left(y^{-}, \varepsilon_{k}\right) \cup U\left(y^{+}, \varepsilon_{k}\right)$. Actually, we only need \#I $\left(y, \varepsilon_{k}\right)<\infty$ for some $y \in J$ since then, for some $k^{\prime}>k$ we obtain \#I $\left(y, \varepsilon_{k}^{\prime}\right)=0$. So, we shall assume \#I $\left(y, \varepsilon_{k}\right)=\infty$ for any $k$ and $y \in A^{+} \cup A^{-}$, for instance take $y \in A^{+}$and we seek for a contradiction. If the set $K:=\left\{n \mid \exists(z, n) \in I\left(y, \varepsilon_{k}\right)\right\}$ is bounded for some $k$ then it is bounded for the same $y$ and any $k^{\prime}>k$. In fact $I\left(y, \varepsilon_{k^{\prime}}\right) \subset I\left(y, \varepsilon_{k}\right)$. Hence, for some $k^{\prime}$ big enough one must have either $I\left(y, \varepsilon_{k}\right)=\emptyset$ or $R_{x}^{-t}(J) \cap J \neq \emptyset$ for some $t>0$, which is a contradiction. Therefore, the set $K$ is unbounded for any $\left(y, \varepsilon_{k}\right)$. As a consequence, for any $y \in A^{+}$ there are sequences $z_{n} \in B_{\varepsilon_{n}}(J)$ and $m_{n} \rightarrow \infty$ such that

$$
R_{x}^{-m_{n}}\left(J\left(z_{n}\right)\right) \cap U\left(y, \varepsilon_{n}\right) \neq \emptyset .
$$

Observe $\left|R_{x}^{-m_{n}}\left(J\left(z_{n}\right)\right)\right| \rightarrow 0$ as $n \rightarrow \infty$; otherwise there is a limit point $z$ of the sequence $\left\{z_{n}\right\}$ whose backward orbit shadows any $z_{n}$ until time $-m_{n}$ and shadows $x$ for the entire past too. Therefore, $\limsup _{t \rightarrow \infty}\left|R_{x}^{-t}\left(W_{\gamma}^{c u}(z)\right)\right|>0$, which is a contradiction. Hence, the sequence $R_{x}^{-m_{n}}\left(z_{n}\right)$ converges to $y \in \Lambda$. Actually, $A^{+} \subset \Lambda$. Notice that if there is some $y \in A^{+} \cap W^{s}(p)$ for some $p \in \operatorname{Per}\left(X^{t}\right)$ we achieve a contradiction again. In fact, in such case, for $\mathrm{k}$ big enough, the set $U\left(y, \varepsilon_{k}\right)$ must be contained in some fundamental domain of $W^{s}(p)$. On the other hand, we can take $\varepsilon_{n_{0}}$ small enough that $\mathcal{O}(p) \cap B_{\varepsilon_{n_{0}}}(J)=\emptyset$. Nevertheless, for $n$ big, $y_{n}=R_{x}^{-m_{n}}\left(J\left(z_{n}\right)\right) \cap W^{s}(p)$ and then $y_{n} \rightarrow \mathcal{O}(p)$ as $n \rightarrow \infty$. 
However,

$$
R_{x_{m_{n}}}^{m_{n}}\left(y_{n}\right) \in R_{x_{-m_{n}}}^{m_{n}}\left(R_{x}^{-m_{n}}\left(J\left(z_{n}\right)\right)\right)=J\left(z_{n}\right) \subset B_{\varepsilon_{n}}(J) \subset B_{\varepsilon_{n_{0}}}(J)
$$

which is a contradiction. Therefore, no point on $A^{+}$belongs to the stable manifold of a periodic point in $\Lambda$.

Take some $y \in A^{+},\left(z_{n}, n\right) \in I\left(y, \varepsilon_{k}\right)$ and $m_{n}>0$, such that for any $w \in J\left(z_{n}\right) \cap \tilde{\Lambda}$ we have

$$
W_{\gamma}^{c s}\left(R_{x}^{-m_{n}}(w)\right) \cap J \subset J^{+} .
$$

Consider a map $\pi: A^{+} \rightarrow A^{+}$defined as follows: For each $z \in A^{+}$, let $w=W_{\gamma}^{c s}(z) \cap$ $J\left(z_{n}\right)$. Then define $\pi(z):=W_{\gamma}^{c s}\left(R_{x}^{-m_{n}}\right)(w) \cap J$. By construction, $\operatorname{dist}\left(R_{x}^{t}(z), R_{x}^{t}(w)\right) \rightarrow$ 0 and $\operatorname{dist}\left(R_{x}^{-t}\left(z_{n}\right), R_{x}^{-t}(w)\right) \rightarrow 0$ as $t \rightarrow \infty$. Then, $w \in \tilde{\Lambda}$ and hence this map is well defined. Moreover, it is continuous and monotone. This implies that there exists $w_{0} \in A^{+} \subset J$ such that $\pi\left(w_{0}\right)=w_{0}$, that is, $R_{x}^{-m_{n}}\left(w_{0}\right)$ belongs to $W_{\gamma}^{c s}\left(w_{0}\right)$. As in the proof of Lemma 5.4 this situation implies that the $\alpha$-limit of $w_{0}$ is a periodic orbit as well for the $\alpha$-limit of $y$. Hence $x$ must be periodic. This contradiction implies that there is $y^{+} \in A^{+}$such that $I\left(y^{+}, \varepsilon_{k}\right)=\emptyset$. Hence, if $x$ is accumulated on both sides of $W_{\gamma}^{c s}(x)$ we are done. If $x$ is accumulated only on one side, the arguments of the proof of Lemma 3.6.1 in [21] apply directly.

We shall refer to those points accumulated only on one side of the box as boundary points of $\tilde{\Lambda}$. Notice that if $B_{\varepsilon}(J)$ is an adapted box, then any sub-box is adapted too. As a consequence of the previous argument, for any non-periodic point $x \in \Lambda$, small adapted boxes can be taken in such a way $\partial^{c u}\left(B_{\varepsilon}(J)\right)$ is contained in some central stable manifolds, unless the point $x$ is a boundary point of $\tilde{\Lambda}$.

Consider an adapted box $B_{\varepsilon}(J)$ associated to some $x \in \Lambda$. If there is $z \in B_{\varepsilon}(J) \cap \Lambda$ such that $z_{-t} \in B_{\varepsilon}(J)(t>0)$ for the first time, we say $\left(\psi_{z}, D_{\psi}\right)$ is a return of $B_{\varepsilon}(J)$ if $D_{\psi}$ is the connected component which contain $z$ of $D_{z}^{-t} \cap B_{\varepsilon}(J)$ and $\psi_{z}=\left.R_{z}^{-t}\right|_{D_{\psi}}$. Notice the holonomy map is defined on some normal section that contains the box. Denote by $\mathcal{R}=\mathcal{R}\left(B_{\varepsilon}(J), \Lambda\right)$ the set of all returns associated to $B_{\varepsilon}(J)$. For $\psi \in \mathcal{R}$ we say $\left|\psi^{\prime}\right|<\xi<1$ if and only if for any $y \in J(z), z \in D_{\psi} \cap \Lambda$ we have that $\left|D R_{z}^{-t}\right|_{\tilde{F}(y)} \mid<\xi$, where $\tilde{F}(y)=T_{y} W_{\gamma}^{c u}(z)$.

Let $\psi \in \mathcal{R}$. If $y \in D_{\psi} \cap \Lambda$ then $J(y) \subset D_{\psi}$; so, it is saturated by central unstable manifolds and hence, is a vertical strip; since any point $\tilde{y} \in J(y)$ shadows $y$ until time $\tau_{z}^{-t}(\tilde{y})$. On the other hand, if $D_{\psi} \subset^{s} B_{\varepsilon}(J)$, then the image $B_{\psi}:=\operatorname{img}(\psi)$ is an horizontal strip, i.e. intersects both components of $\partial^{s} B_{\varepsilon}(J)$, by continuity.

Also we say $\psi>\varphi$ for $\psi, \varphi \in \mathcal{R}$ if $\psi \circ \varphi^{-1}$ is obtained by flowing the orbits a negative amount of time. Remember we are considering first returns for the past.

LEMMA 5.6. - For $\psi_{1}, \psi_{2} \in \mathcal{R}$, if $\psi_{1} \neq \psi_{2}$, then $D_{\psi_{1}} \cap D_{\psi_{2}}$ is not a domain of a return if the intersection does not contains $\partial^{u}\left(B_{\varepsilon}(J)\right)$.

Proof. - Take $\varphi \in \mathcal{R}\left(B_{\varepsilon}(J), \Lambda\right)$ such that $D_{\varphi}=D_{\psi_{1}} \cap D_{\psi_{2}}$. Since $D_{\varphi} \cap \partial^{u} B_{\varepsilon}(J)=\varnothing$ then $B_{\varphi}$ is an horizontal strip. Now $\psi_{1}\left(D_{\varphi}\right) \subset B_{\psi_{1}} \subset B_{\varepsilon}(J)$ since $D_{\varphi} \subset D_{\psi_{1}}$. Since $\varphi$ is a return, there is $z \in D_{\varphi}$ such that $\varphi(z)$ is the first return of $z$ to $B_{\varepsilon}(J)$. Hence $\psi_{1}>\varphi$. 
Observe $\psi_{1} \circ \varphi^{-1}\left(B_{\varepsilon}(J)\right) \subset B_{\varepsilon}(J)$, because it is an adapted box. This is a contradiction to the fact of $\Lambda$ being transitive and not a single periodic orbit.

DEFINITION 6. - An adapted box $B_{\varepsilon}(J)$ is well adapted if there is a sub-box $B_{\varepsilon^{\prime}}(J)$ and two disjoint vertical strips $S_{1}, S_{2}$ such that

$$
B_{\varepsilon}(J) \backslash B_{\varepsilon^{\prime}}(J)=S_{1} \cup S_{2},
$$

each one satisfies either: $S_{i} \cap \Lambda=\emptyset$ or $S_{i}$ is a domain of some $\psi_{i} \in \mathcal{R}\left(B_{\varepsilon}(J), \Lambda\right)$, for which $\psi_{i}\left(S_{i}\right)$ is an horizontal strip.

LEMmA 5.7.- Given any $x \in \Lambda \backslash \operatorname{Per}\left(X^{t}\right)$, there exists well adapted boxes associated to $x$ of arbitrarily small size.

Proof. - Let $B_{\varepsilon}(J)$ be a small adapted box associated to $x \in \Lambda \backslash \operatorname{Per}\left(X^{t}\right)$, for some $J \subset W_{\gamma}^{c u}(x)$. We shall prove there is a sub-box $B_{\varepsilon^{\prime}}(J)$ which is well adapted $\left(\varepsilon^{\prime} \leqslant \varepsilon\right)$. It is important to remark that sub-boxes are not necessarily symmetric with respect to $J$, that is, we shall only need to find an interval $T \subset W_{\varepsilon}^{c s}(x), x \in T$, such that $B_{T}(J)$ have the desired properties. We split the proof in two parts:

First we shall assume that for all $\varepsilon^{\prime} \leqslant \varepsilon$ :

$$
B_{\varepsilon^{\prime}}(J) \neq\left\{y \in J(z) \mid z \in \operatorname{cl}\left(B_{\varepsilon^{\prime}}(J)\right) \cap \Lambda\right\}
$$

For instance, this happens on the right side of the box $B_{\varepsilon^{\prime}}^{+}(J)$. That is, for any $\varepsilon^{\prime} \leqslant \varepsilon$ :

$$
B_{\varepsilon^{\prime}}^{+}(J) \neq\left\{y \in J(z) \mid z \in \operatorname{cl}\left(B_{\varepsilon^{\prime}}^{+}(J)\right) \cap \Lambda\right\} .
$$

So, there is some $w \in W_{\varepsilon}^{c s}(x)$ on the right hand of $x$ such that $w \notin J(z)$ for any $z \in \operatorname{cl}\left(B_{\varepsilon^{\prime}}^{+}(J)\right) \cap \Lambda$. According to this order we have $x<w$ on $W_{\varepsilon}^{c s}(x)$. Define $w_{z}=$ $J(z) \cap W_{\varepsilon}^{c s}(x)$ for any such $z$.

If $w>w_{z}$ for all $z$ then $S_{2}=B_{\varepsilon^{\prime}}^{+}(J) \backslash B_{\left(x, \tilde{w}_{z}\right)}(J)$ is a vertical strip contained $B_{\varepsilon^{\prime}}^{+}(J)$, where $\tilde{w}_{z}=\sup \left\{w_{z} ; z\right\}$ and such that $S_{2} \cap \Lambda=\emptyset$ as the definition requires. Otherwise, there is $z \in \operatorname{cl}\left(B_{\varepsilon^{\prime}}^{+}(J)\right) \cap \Lambda$ such that $w_{z}>w$. Let $w_{0}=\inf \left\{w_{z} \mid w_{z}>w\right\}$. Hence, reducing the right side of the box $B_{\varepsilon^{\prime}}(J)$ to $B_{\left(x, w_{0}\right)}(J)$ we have that $S_{2}=$ $B_{\varepsilon^{\prime}}^{+}(J) \backslash B_{\left(x, \tilde{w}_{z}\right)}(J)$ and $S_{2} \cap \Lambda=\emptyset$.

Now we shall see that the left side of the box also verify (9). In fact, assume by contradiction that for some $\varepsilon^{\prime}>0$

$$
B_{\varepsilon^{\prime}}^{-}(J)=\left\{y \in J(z) \mid z \in \operatorname{cl}\left(B_{\varepsilon^{\prime}}^{-}(J)\right) \cap \Lambda\right\} .
$$

We claim there is $\psi \in \mathcal{R}$ such that

$$
D_{\psi} \cap B_{\varepsilon^{\prime}}^{-}(J) \neq \emptyset \quad \text { and } \quad D_{\psi} \cap\left(\partial^{u} B_{\varepsilon^{\prime}}^{-}(J) \backslash J\right)=\emptyset \text {. }
$$

If the claim holds, then $B_{\psi}$ is an horizontal strip that crosses all $B_{\varepsilon}(J)$ and in particular $B_{\psi} \cap B_{\varepsilon}^{+}(J) \neq \emptyset$. This is a contradiction to (9). Therefore, (9) holds also for the left side of the box. Repeating the argument we have done for the right side on the left side, we obtain the desired sub-box of $B_{\varepsilon}(J)$ which is well adapted. 
To prove the claim, first notice there are $\psi \in \mathcal{R}$ such that $D_{\psi} \cap B_{\varepsilon^{\prime}}^{-}(J) \neq \emptyset$, since there are points of $\Lambda$ on the left side of the box and $\Lambda$ is transitive. If there is only one return, then $B_{\varepsilon^{\prime}}^{-}(J) \subset D_{\psi}$ and also $B_{\psi} \subset\left(B_{\varepsilon^{\prime}}^{-}(J) \backslash J\right)$, since $X^{t}(J) \cap J=\emptyset, \forall t \neq 0$ and also $B_{\psi}$ satisfies (10). This is a contradiction to the transitivity of the set $\Lambda$. Hence, there are at least two returns on the left side of the box.

Suppose there are two different $\psi_{1}, \psi_{2} \in \mathcal{R}$ such that

$$
\left.\left(\partial^{u}\left(B_{\varepsilon^{\prime}}^{-}(J)\right)\right) \backslash J\right) \subset D_{\psi_{1}} \cap D_{\psi_{1}},
$$

$\psi_{1}<\psi_{2}$ and there is no other return preceding $\psi_{2}$ except $\psi_{1}$. Notice $B_{\psi_{1}} \nsubseteq D_{\psi_{1}}$. Moreover, $B_{\psi_{1}} \subset\left(B_{\varepsilon^{\prime}}^{-}(J) \backslash J\right)$, since $B_{\varepsilon}^{+}(J) \cap \Lambda$ have holes as we assumed in (9) and $X^{t}(J) \cap J=\emptyset$ for any $t \neq 0$. Also $D_{\psi_{1}} \subset B_{\varepsilon^{\prime}}^{-}(J)$ since, in the other case $B_{\varepsilon^{\prime}}^{-}(J) \subset D_{\psi_{1}}$ contradicting again the transitivity of $\Lambda$. Notice $D_{\psi_{1}} \subset D_{\psi_{2}}$ since $\psi_{1}<\psi_{2}$ and they are different returns. Therefore there exists $z \in B_{\varepsilon^{\prime}}^{-}(J) \backslash D_{\psi_{1}}$ that returns into $B_{\varepsilon^{\prime}}^{-}(J)$ before $\psi_{2}$ by a continuous extension of $\psi_{1}$. However, $z \in D_{\psi_{2}} \backslash D_{\psi_{1}}$. This contradiction proves our claim.

Now is left to prove the lemma in case that

$$
B_{\varepsilon}(J)=\left\{y \in J(z) \mid z \in \operatorname{cl}\left(B_{\varepsilon}(J)\right) \cap \Lambda\right\} .
$$

Take $\psi_{+}$and $\psi_{-} \in \mathcal{R}$ such that $D_{+} \subset B_{\varepsilon}^{+}(J), D_{-} \subset B_{\varepsilon}^{-}(J)$ and that $B_{\psi_{+}}, B_{\psi_{-}}$ are horizontal strips. It is not difficult to see that we can find two periodic intervals $J\left(w_{+}\right) \subset D_{+}$and $J\left(w_{-}\right) \subset D_{-}$, that is

$$
\psi_{ \pm}\left(J\left(w_{ \pm}\right)\right) \subset J\left(w_{ \pm}\right)
$$

Cutting the box along both intervals we obtain the desired well adapted box, proving the lemma.

\subsection{Proof of Main Lemma}

Let $\Lambda_{0} \subset M$ be a non-trivial compact invariant set provided with a dominated splitting which is not an irrational torus. Assume that any compact invariant subset of $\Lambda_{0}$ is hyperbolic. We shall prove $\Lambda_{0}$ is hyperbolic. In order to do that, we need to prove both: $\left|L^{-t}\right|_{F\left(z_{0}\right)} \mid \rightarrow 0$ and $\left|L^{t}\right|_{E\left(z_{0}\right)} \mid \rightarrow 0$ as $t \rightarrow \infty$ for any $z_{0} \in \Lambda_{0}$. We shall prove only the former limit. The latter is analogous.

First assume $\Lambda_{0}$ is not a minimal set. Recall a compact invariant set is minimal if and only if any orbit is dense. Let us state the following two lemmas. For a proof see [21], Lemma 3.7.4 and Lemma 3.7.2 respectively.

LEMMA 5.8. - Let $B_{\varepsilon}(J)$ be a well adapted box such that \#R( $\left.B_{\varepsilon}(J), \Lambda\right)$ is not bounded. Then, there exists a return $\psi_{0} \in R\left(B_{\varepsilon}(J), \Lambda\right)$ such that the adapted box $B_{\psi_{0}}=\operatorname{Img}\left(\psi_{0}\right)$ satisfies that for every $\psi \in R\left(B_{\psi_{0}}, \Lambda\right)$ we have $\left|\psi^{\prime}\right|<\frac{1}{2}$. 
LEMMA 5.9. - Let $B_{\varepsilon}(J)$ be an adapted box such that for any $\psi \in \mathcal{R}\left(B_{\varepsilon}(J), \Lambda\right)$ we have $\left|\psi^{\prime}\right| \leqslant \xi<1$. Then, for every $y \in B_{\varepsilon}(J) \cap \Lambda_{0}$ we have

$$
\int_{0}^{\infty}\left|R^{-t}(J(y))\right| \mathrm{d} t<\infty
$$

and therefore $\left|D_{y} R^{-t}\right|_{F(y)} \mid \rightarrow 0$ as $t \rightarrow \infty$.

Notice that $\left|D_{y} R^{-t}\right|_{F(y)} \mid \rightarrow 0$ as $t \rightarrow \infty$ implies $\left|L^{-t}\right|_{F(y)} \mid \rightarrow 0$ as $t \rightarrow \infty$.

By hypothesis there is $x \in \Lambda_{0}$ such that $x \notin \omega(x)$. Lemma 5.7 implies the existence of a small well adapted box $B_{\varepsilon}(J)$ associated to $x$ such that $B_{\varepsilon}(J) \cap\left\{X^{-t}(x) \mid t>0\right\}=\emptyset$. Notice $\# R\left(B_{\varepsilon}(J), \Lambda_{0}\right)=\infty$, since $\Lambda_{0}$ is transitive. Therefore combining Lemma 5.8 and Lemma 5.9 we conclude that there exists a sub-box $B_{0}\left(=\operatorname{Img}\left(\psi_{0}\right)\right.$ of the Lemma 5.8) where, for all $y \in B_{0} \cap \Lambda_{0}$ we have $\left|D_{y} R^{-t}\right|_{F(y)} \mid \rightarrow 0$ as $t \rightarrow \infty$, and hence $\left|L^{-t}\right|_{F(y)} \mid \rightarrow 0$ as $t \rightarrow \infty$, as we have remarked above.

Now take $z_{0} \in \Lambda_{0}$, if $\alpha\left(z_{0}\right) \neq \Lambda_{0}$, then by hypothesis it is a hyperbolic set. Therefore $\left|L^{-t}\right| F\left(z_{0}\right) \mid \rightarrow 0$ as $t \rightarrow \infty$. On the other hand, if $\alpha\left(z_{0}\right)=\Lambda_{0}$, then there is $m>0$ such that $z_{-m} \in B_{0}$. Then $\left|L^{-t}\right| F\left(z_{-m}\right) \mid \rightarrow 0$ as $t \rightarrow \infty$, and this is what we wanted to prove.

For the case $\Lambda_{0}$ is minimal, we have to use a different argument. Also we need two lemmas whose proof can be found in [21] (see Lemma 3.7.5 and Lemma 3.7.6).

LEMMA 5.10. - There is $x_{0} \in \Lambda_{0}$ and an adapted box of arbitrarily small size associated to $x_{0}$ such that $B_{\varepsilon}\left(J^{+}\right) \cap \Lambda_{0}=\emptyset$ or $B_{\varepsilon}\left(J^{-}\right) \cap \Lambda_{0}=\emptyset$.

LEMMA 5.11. - Let $B_{\varepsilon}(J)$ be an adapted box such that $B_{\varepsilon}\left(J^{+}\right) \cap \Lambda_{0}=\emptyset$. Then there exists $K>0$ such that $\forall y \in B_{\varepsilon}(J) \cap \Lambda_{0}$

$$
\int_{0}^{\infty}\left|R^{-t}\left(J^{+}(y)\right)\right| \mathrm{d} t<K
$$

and moreover, there is $J_{1}(y), J^{+}(y) \subset J_{1}(y) \subset J(y)$ where both components of $J_{1}(y)-$ $J^{+}(y)$ have length bounded away from zero independently of $y$ and some $\tilde{K}>0$ where

$$
\int_{0}^{\infty}\left|R^{-t}\left(J_{1}(y)\right)\right| \mathrm{d} t<\tilde{K} .
$$

In spite of these, notice that $B=\bigcup_{y \in B_{\varepsilon}(J) \cap \Lambda_{0}} J_{1}(y)$ is a two-dimensional open set contained in some transversal section to the flow where for any $y \in B \cap \Lambda_{0}$ we have:

$$
\int_{0}^{\infty}\left|R^{-t}\left(J_{1}(y)\right)\right| \mathrm{d} t<\tilde{K}
$$

and hence $\left|L^{-t}\right|_{F(y)} \mid \rightarrow 0$ as $t \rightarrow \infty$.

Let $z_{0} \in \Lambda_{0}$. There is some $m>0$ such that $z_{-m} \in B$. Then $\left|L^{-t}\right|_{F\left(z_{-m}\right)} \mid \rightarrow 0$ as $t \rightarrow \infty$. This finishes the proof of the Main Lemma. 


\section{Proof of Theorem A}

Finally, on this section we shall give the proof of Theorem A. For that, consider this two subsets of the space of vector fields, $\mathcal{X}^{1}(M)$ :

$$
\begin{aligned}
& H=\left\{X \in \mathcal{X}^{1}(M) \mid \operatorname{Sing}(X) \cap \operatorname{cl}(\operatorname{Per}(X)) \neq \emptyset\right\} \\
& T g=\left\{X \in \mathcal{X}^{1} \mid X \text { has a homoclinic tangency }\right\} .
\end{aligned}
$$

Define $\mathcal{U}=\mathcal{X}^{1}(M) \backslash \operatorname{cl}(H \cup T g)$ (the closure is on the $C^{1}$-topology). The set $\mathcal{U}$ consist of all vector fields which are not $C^{1}$-approximated by a homoclinic bifurcation. Denote by $\operatorname{Per}_{h}(X)$ the set of hyperbolic periodic orbits of $X^{t}$ of saddle type; we shall prove that for elements in $\mathcal{U}$, the closure of this set has a dominated splitting.

Given $X \in \mathcal{X}^{1}(M)$ and any point $p \in \operatorname{Per}_{h}(X)$, let $\tilde{E}_{p}^{s}(X)$ and $\tilde{E}_{p}^{u}(X)$ be the stable and unstable 2-dimensional subspaces of $T_{p} M$, invariant for the derivative of the flow, respectively. We can define $E_{p}^{s}=\tilde{E}_{p}^{s} \cap \mathcal{N}_{p}$ and $E_{p}^{u}=\tilde{E}_{p}^{u} \cap \mathcal{N}_{p}$, since $X(p) \neq 0$ for any $p \in \operatorname{Per}_{h}(X)$.

Remark 3. - For $p \in \operatorname{Per}_{h}(X)$ we have $L_{p}^{t_{p}}=\left.D_{p} X^{t_{p}}\right|_{\mathcal{N}_{p}}$, where $t_{p}$ denotes the period of $p$. Moreover, $E_{p}^{s}$ and $E_{p}^{u}$ are invariant by $L_{p}^{t_{p}}$ and also $R_{p}^{t_{p}}: N_{p} \rightarrow N_{p}$ is a diffeomorphism. Actually, this is the usual Poincaré first return map on $p$.

The next lemma relates the angle ${ }^{1}$ between the stable and unstable spaces on hyperbolic periodic points of saddle type and the existence of a dominated splitting.

Lemma 6.1. - Let $X \in \mathcal{X}^{1}(M)$. If there exists $\gamma>0$ and a neighborhood $\mathcal{V}(X)$ of $X$ such that for any $Y \in \mathcal{V}(X)$ we have

$$
\operatorname{angle}\left(E_{p}^{s}, E_{p}^{u}\right)>\gamma ; \forall p \in \operatorname{Per}_{h}(Y)
$$

then, $\operatorname{cl}\left(\operatorname{Per}_{h}(X)\right)$ has a dominated splitting.

Proof. - To show the existence of a dominated splitting on $\operatorname{cl}\left(\operatorname{Per}_{h}(X)\right)$ one must proceed to verify that the splitting on $\operatorname{Per}_{h}(X)$ given by the stable and unstable spaces on periodic points is in fact dominated. Then, one can easily extend such splitting to the closure. To see that, we remark that the argument of the proof of Lemma 2.0.1 of [21] relies only on some estimates on the eigenvalues of the derivative on periodic points. In our case, all these estimates remain true for $R_{p}^{t_{p}}$ for each $p \in \operatorname{Per}_{h}(X)$, since each return map is a diffeomorphism (see Remark 3). Finally, in [4] we can see how to perturb the flow in order to obtain the desired Poincaré maps.

Now we can state the fundamental relation between homoclinic tangencies and the existence of a dominated splitting.

THEOREM 6.2. - If $X$ is a Kupka-Smale vector field in $\mathcal{U}$, then the $\operatorname{cl}\left(\operatorname{Per}_{h}(X)\right)$ has a dominated splitting.

It is not difficult to see that the proof of Theorem 6.2 reduces to prove the following lemma:

${ }^{1}$ See [21] for definition. 
LEMMA 6.3. - Let $X \in \mathcal{U}$ be a Kupka-Smale vector field on M. Then there exists a neighborhood $\mathcal{V}(X)$ of $X$ and a number $\gamma>0$ such that $\forall Y \in \mathcal{V}(X)$ and $\forall p \in \operatorname{Per}_{h}(Y)$ we have

$$
\operatorname{angle}\left(E_{p}^{s}(Y), E_{p}^{u}(Y)\right)>\gamma .
$$

Proof. - See the proof of Lemma 2.2.2 in [21], and consider Remark 3.

Let $\Omega(X)$ be the non-wandering set of $X$ and denote by $P_{0}(X)$ and $F_{0}(X)$ the sets of attracting and repelling periodic orbits, respectively. Let $\Omega_{0}(X)=\Omega(X) \backslash\left(P_{0}(X) \cup\right.$ $\left.F_{0}(X) \cup \operatorname{Sing}(X)\right)$.

PROPOSITION 6.4. - There is $\mathcal{U}_{1} \subset \mathcal{U}$ an open and dense set such that for any $Y \in \mathcal{U}_{1}$, $\Omega_{0}(Y)$ is closed and it has a dominated splitting.

Proof. - The function $\Gamma$ that associates to each vector field $X$ the $\operatorname{set} \operatorname{cl}\left(\operatorname{Per}_{h}(X)\right)$ is lower semi-continuous, since hyperbolic periodic orbits cannot be destroyed by small perturbations.

Call $G_{1}$ the resiudal set of continuity points of $\Gamma$. Denote by $G_{2}$ the set of KupkaSmale flows on $M$ (i.e. periodic orbits and singularities are hyperbolic, and the stable and unstable manifolds are in general position) and set $G_{3}=\{X \mid \Omega(X)=\operatorname{cl}(\operatorname{Per}(X)) \cup$ $\operatorname{Sing}(X)\}$, which is also residual (see [19]). Hence, $G=G_{1} \cap G_{2} \cap G_{3}$ is a residual set.

LEMMA 6.5. - For any $X \in G \cap \mathcal{U}$, we have $\Omega_{0}(X)=\Gamma(X)$.

Proof. - In order to see that $\Omega_{0}(X) \subset \Gamma(X)$, suppose there is $q \in \Omega_{0}(X) \backslash \Gamma(X)$. Let $U$ be an open set such that $\Gamma(X) \subset U$ such that $q \notin \operatorname{cl}(U)$. Let $N \subset \mathcal{U}$ be a neighborhood of $X$ where $\Gamma(Y) \subset U$ for any $Y \in N$. Since $X \in G_{3}$, there is a sequence $\left\{p_{n}\right\} \subset \operatorname{Per}(X) \cup \operatorname{Sing}(X)$ such that $p_{n} \notin \operatorname{cl}(U)$ and $p_{n} \rightarrow q$. Since $\# \operatorname{Sing}(X)<\infty$ and $q \notin \Gamma(X)$ then $p_{n} \in P_{0}(X) \cup F_{0}(X)$ for all $n$. Without lost of generality we may think $p_{n} \in P_{0}(X)$ for all $n$. Let us recall the following theorem due to Pliss.

THEOREM 6.6 (Pliss, [18]). - Let $X \in \mathcal{X}^{1}(M)$ and suppose there is an infinite sequence $\left\{p^{n}\right\} \subset P_{0}(X)$, then for any $\varepsilon>0$ there is $Y \in \mathcal{X}^{1}(M), \varepsilon-C^{1}$-close to $X$ and some $n \in \mathbb{N}$ such that $p_{n}$ is a non-hyperbolic periodic orbit of $Y$.

It is not difficult to see that if we take a very small perturbation of the map $Y$, we can assume $p_{n}$ is an hyperbolic periodic orbit of saddle type. Hence we have found some $Y \in N$ with a hyperbolic periodic orbit of saddle type $p_{n}$ outside $U$; contradicting the fact of $\Gamma(Y) \subset U$. Therefore $\Omega_{0}(X) \subset \Gamma(X)$. On the other hand, to prove $\Gamma(X) \subset \Omega_{0}(X)$ we claim that $\Omega_{0}(X)$ is closed. If it is not the case, it happens that $\operatorname{cl}\left(\Omega_{0}(X)\right) \cap \operatorname{Sing}(X) \neq \emptyset$. Since $\operatorname{cl}\left(\Omega_{0}(X)\right) \subset \Gamma(X)$, we have found a contradiction to the fact that $\Gamma(X) \cap \operatorname{Sing}(X)=\emptyset$, proving $\Omega_{0}(X)$ is a closed set and hence the lemma follows.

Since $X \in G \cap \mathcal{U}$, we have $\Omega_{0}(X)=\operatorname{cl}\left(\operatorname{Per}_{h}(X)\right)$. As a consequence of Theorem 6.2 we know that $\Omega_{0}(X)$ has a dominated splitting. Take an admissible neighborhood $U$ of $\Omega_{0}(X)$, that is, a neighborhood $U$ such that there is $\mathcal{U}(X)$ a neighborhood of $X$ such that for any $Y \in \mathcal{U}(X)$ the maximal invariant set of $Y$ in $U$ also has a dominated splitting. We can assume $\mathcal{U}(X) \subset \mathcal{U}$. Since $X \in G_{1}$ it is a continuity point of $\Gamma$, given a neighborhood 
$U_{0}$ of $\Omega_{0}(X)$ such that $U_{0} \subset \operatorname{cl}\left(U_{0}\right) \subset U$, we can assume that for all $Y \in \mathcal{U}(X)$ verify that $\operatorname{cl}\left(\operatorname{Per}_{h}(Y)\right) \subset U_{0}$.

Let us prove that for all $Y \in \mathcal{U}(X)$ we have $\Omega_{0}(Y) \subset U$. If it is not the case, there is $Y \in \mathcal{U}(X)$ and $x \in \Omega_{0}(Y) \cap(M \backslash U)$. Notice $x$ is not a periodic point and is nonwandering. Moreover, $x$ cannot be accumulated by points in $P_{0}(Y) \cup F_{0}(Y)$, otherwise, as in the proof of the Lemma 6.5, we can find an hyperbolic periodic orbit of saddle type outside $U_{0}$, which is impossible.

Now the $C^{1}$-Closing Lemma [19] allow us to find $\tilde{Y}$ close to $Y$ having a periodic orbit passing through $x$. Actually, perturbing a bit more if necessary, this orbit is hyperbolic of saddle type. This is a contradiction to the continuity of $\Gamma$ on $X$. Hence, for all $Y \in \mathcal{U}(X)$, the set $\Omega_{0}(Y)$ has a dominated splitting. Finally, the set $\mathcal{U}_{1}=\bigcup_{X \in G} \mathcal{U}(X)$ verifies the proposition.

Now we can give a proof of Theorem A.

Proof of Theorem A. - Given $X \in \mathcal{U}$, Proposition 6.4, together with Theorem B imply there is a $C^{2}$-Kupka-Smale vector field $Y \in \mathcal{U}_{1}$ arbitrarily close to $X$ such that $\Omega_{0}(Y)=\Lambda \cup \mathcal{T}$, where $\Lambda$ is hyperbolic and $\mathcal{T}$ is a finite union of irrational torus. However, the existence of such torus is not generic, even in the $C^{2}$ topology. Hence, making a small perturbation if necessary, we obtain that $\Omega_{0}(Y)$ is hyperbolic.

Now we claim $\Omega(Y)$ is hyperbolic. For that, we just need to guarantee that $P_{0}(Y) \cup$ $F_{0}(Y)$ is a finite set. In fact, for instance, suppose $\# P_{0}(Y)=\infty$. Let $U$ be a neighborhood of $\Omega_{0}(Y)$ where the maximal invariant set on $U$ is hyperbolic. Notice we can take $U$ in such a way all periodic orbits in $U$ are of saddle type. On the other hand, all but a finite number of periodic attracting orbits must belong to $U$. In fact, $\operatorname{cl}\left(P_{0}(Y)\right) \backslash$ $P_{0}(Y) \subset \Omega_{0}(Y) \subset U$, by definition. This is a contradiction. Therefore $\# P_{0}(Y)<\infty$. This argument applies also to $F_{0}(Y)$. Hence, we conclude $\Omega(Y)$ is hyperbolic.

It is left to prove $\Omega(Y)=\operatorname{cl}(\operatorname{Per}(X))$ and that there are no cycles. Since $L(Y) \subset \Omega(Y)$, the limit set of $Y$ is hyperbolic too and so, the periodic orbits are dense on it. By a result of Newhouse [11], we only need to verify the absence of cycles on $L(Y)$ in order to conclude $L(Y)=\Omega(Y)$. Since there are no singularities of the flow involved, the index of all saddle periodic orbits is one. And since $Y$ is Kupka-Smale vector field, all the invariant manifolds are in general position. Hence there are no cycles.

Now, we have already prove that $X$ can be approximated by $Y$ either: Uniformly hyperbolic with no-cycle condition, exhibiting a homoclinic tangency, or $X$ belongs to the class $H$. However, if $X \in H$, it is an easy consequence of Hayashi's Connecting Lemma [4] that there is $Y \in \mathcal{X}^{1}(M)$ arbitrarily close to $X$ showing a singular cycle, concluding the proof of Theorem A.

\section{Acknowledgements}

This is the first author's doctoral thesis, under the orientation of Jacob Palis at IMPA, and he is very thankful for his encouragement and guidance. 


\section{REFERENCES}

[1] C. Bonatti, M. Viana, SRB measures for partially hyperbolic dynamical systems whose central direction is mostly contracting, Israel J. Math. 115 (2000) 157-193.

[2] C.I. Doering, Persistently transitive vector fields on three manifolds, in: Dynam. Syst. Biff. Theory, Pitman Res. Notes, Vol. 160, 59-89.

[3] J. Guckenheimer, R.F. Williams, Structural stability of Lorenz attractors, Inst. Hautes Études Sci. Publ. Math. 50 (1979) 59-72.

[4] S. Hayashi, Connecting invariant manifolds and the solution of the $C^{1}$ stability and $\Omega$-stability conjectures for flows, Ann. of Math. (2) 145 (1) (1997) 81-137.

[5] M.-R. Herman, Sur la conjugaison différentiable des difféomorphismes du cercle à des rotations, Inst. Hautes Études Sci. Publ. Math. 49 (1979) 5-233.

[6] M.W. Hirsch, C.C. Pugh, M. Shub, in: Invariant Manifolds, Lecture Notes in Math., Vol. 583, Springer-Verlag, Berlin, 1977.

[7] R. Labarca, M.J. Pacífico, Stability of singularity horseshoes, Topology 25 (3) (1986) 337352.

[8] R. Mañé, Ergodic Theory and Differential Dynamics, Springer-Verlag, New York, 1987.

[9] C.A. Morales, M.J. Pacífico, E. Pujals, On $C^{1}$ robust singular transitive sets for threedimensional flows, C. R. Acad. Sci. Paris Sér. I Math. 326 (1) (1998) 81-86.

[10] S. Newhouse, Diffeomorphisms with infinitely many sinks, Topology 13 (1974) 9-18.

[11] S. Newhouse, Hyperbolic Limit Sets, Trans. Amer. Math. Soc. 167 (1972) 125-150.

[12] S. Newhouse, Lectures on dynamical systems, in: Dynamical Systems, CIME Summer School, Bressanone, 1978, in: Progr. Math., Vol. 8, Birkhäuser, Boston, MA, 1980, pp. 1114.

[13] V.I. Oseledets, A multiplicative ergodic theorem: Lyapunov characteristic numbers for dynamical systems, Trans. Moscow Math. Soc. 19 (1968) 197-231.

[14] J. Palis, A global view of dynamics and a conjecture on the denseness of finitude of attractors, Asterisque 261 (2000) 339-351.

[15] J. Palis, On Morse-Smale dynamical systems, Topology 8 (1968) 385-404.

[16] J. Palis, S. Smale, Structural stability theorems, Proc. Amer. Math. Soc. Symp. Pure Math. 14 (1970) 223-232.

[17] J. Palis, F. Takens, Hyperbolicity and Sensitive Chaotic Dynamics of Homoclinic Bifurcations, Cambridge Univ. Press, Cambridge, 1993.

[18] V.A. Pliss, On a Conjecture of Smale, Differentsial'nye Uravneniya 8 (1972) 268-282.

[19] C. Pugh, The closing lemma, Amer. J. Math. 89 (1967) 956-1009.

[20] C. Pugh, An improved closing lemma and a general density theorem, Amer. J. Math. 89 (1967) 1010-1021.

[21] E. Pujals, M. Sambarino, Homoclinic tangencies and hyperbolicity for surface diffeomorphisms, Ann. of Math. (2) 151 (3) (2000) 961-1023.

[22] E. Pujals, M. Sambarino, On the dynamics of dominated splitting, to appear.

[23] A.J. Schwartz, A generalization of a Poincaré-Bendixon theorem to closed two dimensional manifolds, Amer. J. Math. 85 (1963) 453-458; Errata, ibid 85 (1963) 753.

[24] S. Smale, Differentiable dynamical systems, Bull. Amer. Math. Soc. 73 (1967) 747-817.

[25] A. Tahzibi, Stably ergodic systems which are not partially hyperbolic, to appear. 